\title{
UNIVERSITY COLLEGE CARDIFF RADIOCARBON DATES I
}

QUENTIN DRESSER

\author{
Radiocarbon Dating Laboratory, Department of Plant Science \\ University College, Cardiff, Wales
}

The laboratory was established by University College, Cardiff, in 1974 primarily for research in vegetation history and archaeology in Britain. The laboratory has been supported yearly since 1978 by the Conservation and Land Division of the Welsh Office, Cardiff, which submits archaeologic samples from rescue excavations in Wales which are carried out by the Clwyd-Powys Archaeological Trust Ltd (CPAT), the Dyfed Archaeological Trust Ltd (DAT), the Glamorgan Gwent Archaeological Trust Ltd (GGAT), and the Gwynedd Archaeological Trust Ltd (GAT).

The method employed is gas proportional counting of synthetic methane. After initial sorting and possible treatment with $5 \% \mathrm{H}_{2} \mathrm{O}_{2}$ to break up clay, charcoal samples are washed with $2.5 \% \mathrm{HCl}$ followed by $2.5 \% \mathrm{NaOH}$. The sample is then placed in a soxhlet extractor to remove humic substances. The charcoal is acidified and the soxhlet extraction continued for a further period before drying.

Bone pretreatments comprise extraction of collagen. After initial washing, the sample is demineralized in $5 \% \mathrm{HCl}$. The solid residue is macerated and suspended in $2.5 \% \mathrm{NaOH}$ followed by suspension in $2.5 \% \mathrm{HCl}$ and then distilled water. The suspension is boiled to solubilize the collagen which is then filtered and evaporated to dryness.

Peat pretreatments are designed to provide a physico-chemical fraction which is most likely to be contemporary with the pollen (Dresser, 1970). Unless otherwise stated, peat pretreatments comprise suspension of the sample in $2.5 \% \mathrm{HCl}$ followed by $2.5 \% \mathrm{NaOH}$. After several washes, the sample is acidified and filtered through a $250 \mu \mathrm{m}$ mesh to remove rootlets. The fine insoluble residue is washed and dried. Inorganic reagents and distilled water are used in all pretreatments.

Samples are combusted in a "Phonon" unit (Switsur, 1972) or occasionally by the tube method for samples with low $\mathrm{C}$ content. The $\mathrm{CO}_{2}$ is purified by bubbling through $\mathrm{KMnO}_{4}$ solution and passage over $\mathrm{Cu} / \mathrm{CuO}$ at $620^{\circ} \mathrm{C}$. The $\mathrm{CO}_{2}$ is converted to $\mathrm{CH}_{4}$ in a closed circuit system. $\mathrm{H}_{2}$ is bled into the system through an electronically controlled needle-valve, and a pump circulates the gas mixture over a ruthenium catalyst at $520^{\circ} \mathrm{C}$. Within the circuit is a water trap cooled by solid $\mathrm{CO}_{2}$. The conversion is completed in $<1.5 \mathrm{~h}$ and there is no detectable residual $\mathrm{CO}_{2}$. All samples are stored for $>1$ month in gas form before counting to allow radon to decay. The detector is filled through a catalytic deoxygenator containing palladium. The fill pressure of ca $2.5 \mathrm{~b}$ is measured using a high accuracy pressure transducer and is temperature compensated.

The detector, manufactured at the Atomic Energy Research Establishment, Harwell, is a $2 \mathrm{~L}$ OFHC copper tube with teflon insulators and a $12.5 \mu \mathrm{m}$ tungsten wire anode. It is surrounded by a multianode gas flow guard detector, also manufactured at AERE Harwell. The detector assembly is shielded by $>8 \mathrm{~cm}$ of boron loaded plastic and $>23 \mathrm{~cm}$ of steel 
plate weighing $8 \mathrm{t}$ from a gasometer constructed in 1936. The laboratory is situated in the basement of a 3-story building. Commercial charge sensitive preamplifiers are used and the main amplifiers and logic circuitry are NIM modules. A custom-built scaler counts $\beta, \alpha$, gross, and guard pulses, and data is printed out every 72 minutes. In addition, a noise channel is counted. A custom-built unit after a design by Arnow (1977), outputs a pulse when $\beta$ pulses occur within $1 \mathrm{~ms}$. It outputs pulses at a predictable low frequency when the source is truly random but at a higher frequency when non-random noise is present.

The background is ca $4.4 \mathrm{cpm}$ and is subject to a barometric effect of $0.18 \% / \mathrm{mb}$. The gross and guard counts are similarly affected and are also subject to diurnal variation and a longer term seasonal variation, though no such variation is observed in the background. The modern reference material used is NBS oxalic acid (SRM 4990-B), which is prepared by wet oxidation. Once in the counter, the gas is subject to a slight quenching effect probably due to outgassing of the teflon insulators. The condition of the gas is fully restored by passage through the palladium catalyst, as determined by channels ratio whilst monitoring an external ${ }^{137} \mathrm{Cs}$ source.

$\delta^{13} \mathrm{C}$ measurements are made on $\mathrm{CO}_{2}$ sub-samples. All sample activities are normalized to $-25 \%$ and the standards to $-19 \%$ (PDB). Date calculations are made using the Libby half-life of $5568 \mathrm{yr}$ and 0.95 of the NBS oxalic activity (ca $25 \mathrm{cpm})$. The $1 \sigma$ quoted error is derived from all experimental errors and incorporates counting statistics and uncertainties in the fill pressure, barometric correction, quench correction, and $\delta^{13} \mathrm{C}$ assay. No term is included to cover sample integrity or whole system repeatability. Small samples are diluted and the error term then includes uncertainty in the dilution factor. Such samples are counted for a longer time than the normal $23 \mathrm{hr}$. The laboratory participated in the Harwell intercalibration experiment (Otlet et al, 1980).

\section{ACKNOWLEDGMENTS}

The author thanks R L Otlet for assistance in the supply of the detectors, H Tauber and R Burleigh for the supply of check samples, and G Hillman for making charcoal identifications. Grateful thanks to the Welsh Office for financial support and to submitters who supplied details enabling these reports to be written. For technical assistance throughout the laboratory thanks are due to $\mathrm{S}$ Willis and $\mathrm{K}$ Polniaszek; the latter also helped in the assembly of this report. Finally, thanks are due to A G Smith who founded the laboratory and made it possible.

\section{CHECK SAMPLES}

\section{CAR-2. 1830 to 1840 oak wood}

$100 \pm 80$

$\delta^{13} C=-25.8 \%$

Sample from tree felled 1970 at Townley Hall, $5.5 \mathrm{~km} \mathrm{~W}$ of Drogheda, Co Louth, Ireland ( $53^{\circ} 43^{\prime} \mathrm{N}, 6^{\circ} 27^{\prime} \mathrm{W}$, Natl Grid Ref O 035765). Coll by $\mathrm{J}$ Hood, Dept Botany, Trinity Coll, Dublin. Result is mean and standard deviation of 5 counts $(60 \pm 50,230 \pm 60,80 \pm 60,70 \pm 55,50 \pm 55)$. Comment: similar sample dated at Dublin lab (R, 1974, v 16, p 6). 
CAR-3. Ruds Vedby

$$
\begin{array}{r}
11,030 \pm 100 \\
\delta^{13} C=-26.9 \%
\end{array}
$$

Wood from dark layer representing pollen zone Boundary II/III, isolated from peaty lake mud in profile at Ruds Vedby, Zealand, Denmark $\left(55^{\circ} 32^{\prime} \mathrm{N}, 11^{\circ} 22^{\prime} \mathrm{E}\right)$. Coll 1951 by H Krog. Comment: sample dated extensively as check sample and summarized by Håkansson (R, 1968, v 10, p 38) who gives mean of previous measurements of 10,995 \pm 55 .

\section{CAR-4. Tjanefer}

$\mathbf{3 0 1 0} \pm \mathbf{5 0}$

$$
\delta^{13} \mathrm{C}=-25.7 \%
$$

Wood from branch embedded during construction in mud-brick superstructure of tomb of Tjanefer at Dra' Abu el-Naga', Thebes, Egypt $\left(25^{\circ} 41^{\prime} \mathrm{N}, 32^{\circ} 40^{\prime} \mathrm{E}\right)$. Coll 1967 by G T Martin. Comment: dated by British Mus lab to $3080 \pm 75$ (BM-337, R, 1971, v 13, p 162).

\section{CAR-5. Mount Pleasant}

$3890 \pm 60$

$\delta^{13} C=-24.7 \%$

Charcoal from secondary silts of ditch surrounding timber structure at late Neolithic henge monument at Mt Pleasant, Dorchester, Dorset, England (50 $42^{\prime} \mathrm{N}, 2^{\circ} 24^{\prime} \mathrm{W}$, Natl Grid Ref SY 710900). Coll 1970 by G J Wainwright. Comment: dated by British Mus lab to $3630 \pm 60$ (BM-668, R, 1976, v 18, p 23).

\section{GEOLOGIC SAMPLES}

\section{Wales}

\section{Ardudwy peat series}

Peat samples are from excavated trench $180 \mathrm{~m} \mathrm{~N}$ of Moel-y-Gerddi archaeol site at Ardudwy, 3.5km ENE of Harlech, Gwynedd $\left(52^{\circ} 52^{\prime} \mathrm{N}, 4^{\circ}\right.$ $03^{\prime}$ W, Natl Grid Ref SH 616319). Single peat monolith was coll 1980 by $\mathrm{R}$ Kelly, GAT, and was investigated palynologically by $\mathrm{S} M$ Price and F M Chambers, Dept Geog, Univ Keele, Staffs. Subm by GAT. Dates were required for determination of accumulation-rate in conjunction with absolute pollen diagram. Comments refer to relative pollen data (based on sum of total land pollen).

CAR-660. $17-19 \mathrm{~cm}$

$1670 \pm 60$

$\delta^{13} C=-28.9 \%$

Peat at decline of arboreal pollen from $51 \%$ at $18 \mathrm{~cm}$ to $35 \%$ at $16 \mathrm{~cm}$.

CAR-641. $\quad 42-44 \mathrm{~cm}$

$3720 \pm 70$

$\delta^{13} \mathrm{C}=-28.8 \%$ o $40 \mathrm{~cm}$.

Peat at start of decline in arboreal pollen from $90 \%$ at $44 \mathrm{~cm}$ to $60 \%$ at

CAR-661. 67-69cm

$4730 \pm 80$

$\delta^{13} C=-29.4 \%$

Peat at decline of arboreal pollen from $74 \%$ at $68 \mathrm{~cm}$ to $49 \%$ at $66 \mathrm{~cm}$. 
CAR-642. 80-82cm

$5200 \pm 80$ pollen at $78 \mathrm{~cm}$.

CAR-662. 99-101 cm

Peat with arboreal pollen at $95 \%$.

CAR-663. $\quad 109-111 \mathrm{~cm}$

Peat with arboreal pollen at $90 \%$.

CAR-664. 129-131 cm

Peat at peak in Alnus pollen at $84 \%$.

CAR-643. 138-140cm

Basal layer of peat. Alnus pollen at $5 \%$. $\mathbf{5 7 1 0} \pm \mathbf{8 0}$

$\delta^{13} C=-28.8 \%$

$6440 \pm 90$

$\delta^{13} \mathrm{C}=-28.9 \%$

$7510 \pm 100$

$\delta^{13} C=-29.2 \%$

$8590 \pm 100$

$\delta^{13} C=-29.8 \%$

General Comment (FMC): confirmation of early Alnus in NW Wales given by CAR-643 and -664.

\section{Cefn Graeanog Bog series}

Peat samples are from monolith of mire sediments taken in conjunction with archaeol excavations of Romano-British hut group and medieval farmstead (Kelly, 1982) at Cefn Graeanog, $14 \mathrm{~km} \mathrm{~S}$ of Caernavon, Gwynedd $\left(53^{\circ} 01^{\prime} \mathrm{N}, 4^{\circ} 18^{\prime} \mathrm{W}\right.$, Natl Grid Ref SH 453487). Coll 1977 by M Girling and subm by GAT. Pretreatment and pollen analysis by F M Chambers (1980; 1983a), Dept Plant Sci, Univ Coll, Cardiff. Comment (FMC): $20 \times 20 \mathrm{~cm}$ monolith of depth $213 \mathrm{~cm}$ was taken ca $200 \mathrm{~m}$ W of Cefn Graeanog Hut Group II, in valley mire. Stratigraphy comprised 0 to $10 \mathrm{~cm}$ upcast, 10 to $60 \mathrm{~cm}$ valley bog peat, 65 to $206 \mathrm{~cm}$ carr peat, and 206 to $213 \mathrm{~cm}$ bluish gray silt. Sample depths refer to present surface. Dates required for determination of deposition-rate curve for detailed pollen diagram.

CAR-67. CGB $35-37 \mathrm{~cm}$

Peat.

CAR-68. CGB 44-46cm

Peat.

CAR-72. CGB 50-51 cm

Peat. Comment (FMC): too young.

CAR-69. CGB 57-58cm

Peat. Comment (FMC): too young. $\mathbf{7 5 0} \pm \mathbf{4 0}$

$\delta^{13} \mathrm{C}=-28.0 \%$

$1230 \pm 40$

$\delta^{13} C=-28.5 \%$

$1130 \pm 50$

$\delta^{13} C=-28.6 \%$

$1040 \pm 50$

$\delta^{13} C=-28.5 \%$ 
CAR-70. CGB 65-66cm

$\mathbf{2 3 1 0} \pm \mathbf{4 0}$

$\delta^{13} C=-28.4 \%$

Peat from upper horizon of carr peat.

CAR-108. CGB 116-118cm

$\delta^{13} C=-29.2 \%$

Peat from ca $10 \mathrm{~cm}$ above elm decline.

\section{CAR-109. CGB 164-166cm}

Peat.

CAR-110. CGB 202-204cm

$\mathbf{5 6 0 0} \pm \mathbf{7 0}$

$\delta^{13} C=-29.1 \%$ pollen.

Peat from base of valley mire. Alnus values of $30 \%$ to $40 \%$ of total land

General Comment (FMC): results fit relatively smooth deposition-rate curve except for CAR-69 and -72 which appear young.

\section{Breidden Pond series}

Organic mud samples are from 2 monoliths taken from natural pond within Iron age hillfort excavated by $\mathrm{C} \mathrm{R}$ Musson and W J Britnell, CPAT, at Breidden Mt, $10 \mathrm{~km}$ NE of Welshpool, Powys $\left(52^{\circ} 43^{\prime} \mathrm{N}, 3^{\circ} 03^{\prime} \mathrm{W}\right.$, Natl Grid Ref SJ 295145). Pollen analysis carried out by C A Green and A G Smith, Dept Plant Sci, Univ Coll, Cardiff. Coll 1976 by AGS and WJB and subm by CPAT. Layers B to $\mathrm{H}$ refer to strat sub-units (organic muds and silts) established by excavators. Comment: all samples were normal fine fractions and combusted in tube. Depths refer to modern surface.

\section{CAR-382. Br I, 40-41 cm}

$1020 \pm 60$

Brown organic mud near base of Layer B.

\section{CAR-137. Br I, 44-45cm}

$$
\begin{array}{r}
\mathbf{2 2 8 0} \pm \mathbf{6 0} \\
\delta^{13} C=-30.3 \% \text { o }
\end{array}
$$

Gray-brown clayey silt at top of Layer C. Comment (AGS): comparison with CAR-382 indicates unconformity between Layers C and B. Conclusion is supported by pollen evidence.

CAR-383. Br I, 45-46cm

Gray-brown clayey silt from top of Layer C.

CAR-138. Br I, 49-50cm

Gray-brown clayey silt from Layer C.

$$
\begin{array}{r}
\mathbf{2 2 1 0} \pm \mathbf{6 0} \\
\delta^{13} C=-30.2 \% 0
\end{array}
$$

$2390 \pm 60$

$\delta^{13} C=-30.4 \%$ 
CAR-381. Br I, 54-55cm

$2440 \pm 70$

Gray-brown clayey silt from Layer C.

CAR-139. Br I, 56-57cm

$2810 \pm 60$

Gray-brown clayey silt from Layer C, at beginning of decline of tree pollen. Comment: ${ }^{13} \mathrm{C}$ value of $-30 \% 0 \pm 0.5$ used (mean and standard deviation of other samples in series).

CAR-140. Br I, 62-63cm

Gray-brown clayey silt near base of Layer C.

\section{CAR-141. Br I, 67-68cm}

Dark brown organic mud from top of Layer D.

\section{CAR-142. Br I, 71-73cm}

Dark brown organic mud from Layer D.

\section{CAR-143. Br I, 82-84cm}

$$
\delta^{13} C=\begin{array}{r}
\mathbf{2 9 7 0} \pm \mathbf{6 0} \\
-29.7 \% 0
\end{array}
$$

$$
\begin{array}{r}
3150 \pm 70 \\
\delta^{13} C=-30.1 \%
\end{array}
$$

$3250 \pm 70$

$\delta^{13} C=-30.0 \%$

$4420 \pm 70$

$\delta^{13} C=-30.1 \%$

Dark brown organic mud from Layer D, at decline of Quercus and Ulmus pollen curves.

\section{CAR-157. Br I, $109-110 \mathrm{~cm}$}

$6900 \pm 90$

Dark brown clay-mud from Layer E, at rise of Alnus pollen curve.

\section{CAR-158. Br I, $124-125 \mathrm{~cm}$}

Dark brown clayey silt from Layer F.
$8510 \pm 100$

$\delta^{13} C=-27.5 \%$

$\mathbf{1 4 , 5 6 0} \pm 210$

$\delta^{13} C=-26.1 \%$

CAR-159. Br II, $150-152 \mathrm{~cm}$

Dark brown clayey silt from Layer H. Comment (AGS): remnant portion of truncated Layer $\mathrm{H}$ belongs to earliest part of Windermere Interstadial as SRR-681, 14,560 \pm 280 (R, 1981, v 23, p 268; Pennington, 1977). Pollen evidence shows open plant community.

\section{Pen Rhiw Wen series}

Peat samples are from series of 5 monoliths and 1 core in varying depths of blanket peat on several substrates all within $1 \mathrm{~km}^{2}$ at Pen Rhiw Wen, $4.5 \mathrm{~km} \mathrm{~N}$ of Brynamman, Dyfed $\left(51^{\circ} 50^{\prime} \mathrm{N}, 3^{\circ} 50^{\prime} \mathrm{W}\right.$, Natl Grid Ref SN 732183). Coll and subm 1977 by E Cloutman, Dept Plant Sci, Univ Coll, Cardiff. Comment (EC): palynol studies were made to obtain 3-dimensional appraisal of development of peat and surrounding vegetation (Cloutman, 
1983). Monolith $\mathrm{H}$ was taken in one $90 \mathrm{~cm}$ sec from blanket peat over conglomerate at alt $510 \mathrm{~m}$. Core J was taken in 3 secs from valley bog in swallow hole on conglomerate at alt $490 \mathrm{~m}$. Length of core, $4.7 \mathrm{~m}$. Monolith $\mathrm{K}$ was taken in 2 secs from blanket peat overlying podsolic soil on $4^{\circ}$ slope on conglomerate at alt $495 \mathrm{~m}$. Length of monolith, $1.29 \mathrm{~m}$. Monolith $\mathrm{L}$ was taken in one $66 \mathrm{~cm}$ sec from blanket peat overlying iron pan podsol with surface gleying on $11^{\circ}$ slope immediately below Site $\mathrm{M}$ at alt $512 \mathrm{~m}$. Monolith $\mathrm{M}$ was taken in one $76 \mathrm{~cm}$ sec from blanket peat overlying iron pan with surface gleying on $6^{\circ}$ slope at alt $540 \mathrm{~m}$. Monolith $\mathrm{N}$ was taken in one $44 \mathrm{~cm} \mathrm{sec}$ from shallow blanket peat directly over conglomerate ca $30 \mathrm{~m}$ from cairn of Garreg-lwyd Mt at alt $600 \mathrm{~m}$. All samples are fine fraction of peat.

CAR-21. Monolith H $11 \mathrm{~cm}$

$780 \pm 60$

Ombrogenous peat with high Gramineae and Plantago pollen values.

CAR-22. Monolith $\mathrm{H} 26 \mathrm{~cm}$

$1250 \pm 60$

$\delta^{13} C=-28.2 \%$

Ombrogenous peat at increase in Corylus and other tree pollen values.

CAR-23. Monolith $\mathrm{H} 49 \mathrm{~cm}$

$2100 \pm 70$

Ombrogenous peat at rise in Plantago and Gramineae pollen, with high Calluna pollen values.

CAR-24. Monolith $\mathrm{H} 69 \mathrm{~cm}$

$2750 \pm 70$

Ombrogenous peat at start of rise in Calluna pollen value.

CAR-25. Monolith H $79 \mathrm{~cm}$

$2970 \pm 80$

$\delta^{13} C=-28.7 \%$

Ombrogenous peat at fall in Corylus and rise in Cyperaceae pollen values.

CAR-26. Monolith $\mathrm{H} 87 \mathrm{~cm}$

$3380 \pm 70$

Ombrogenous peat directly above mineral soil.

$\delta^{13} \mathrm{C}=-28.8 \%$

CAR-28. Core J $22 \mathrm{~cm}$

$890 \pm 70$

$\delta^{13} \mathrm{C}=-27.1 \%$

Eriophorum peat with high Gramineae and Corylus pollen values.

CAR-29. Core J $69 \mathrm{~cm}$

$990 \pm 70$

$\delta^{13} \mathrm{C}=-27.1 \%$

Ombrogenous peat at increasing Gramineae, Plantago and Cyperaceae pollen and declining Betula pollen values. 
CAR-30. Core J $173 \mathrm{~cm}$

$\mathbf{2 4 0 0} \pm \mathbf{5 0}$

Ombrogenous peat at rising Corylus pollen value.

CAR-31. Core J $284 \mathrm{~cm}$

Ombrogenous peat at rising Corylus pollen value but decline in other tree spp pollen.

CAR-32. Core J $370 \mathrm{~cm}$

$3150 \pm 90$

Ombrogenous peat with Calluna fragments.

$$
\delta^{13} C=-27.8 \%
$$

\section{CAR-33. Monolith K $15 \mathrm{~cm}$}

$$
\delta^{13} C=\begin{array}{r}
\mathbf{5 3 0} \pm \mathbf{8 0} \\
-28.3 \%
\end{array}
$$

Ombrogenous peat with high Calluna and Gramineae pollen values.

\section{CAR-34. Monolith K $37 \mathrm{~cm}$}

$$
\begin{array}{r}
\mathbf{2 0 6 0} \pm \mathbf{7 0} \\
\delta^{13} C=-27.5 \% 0
\end{array}
$$

Ombrogenous peat at rise in Calluna and Plantago pollen values.

\section{CAR-35. Monolith $\mathrm{K} 77 \mathrm{~cm}$}

$$
\begin{array}{r}
\mathbf{3 1 3 0} \pm \mathbf{6 0} \\
\delta^{13} C=-27.5 \% 0
\end{array}
$$

Ombrogenous peat at rise in Betula and Quercus pollen and decline in Pteridium and Corylus pollen values.

\section{CAR-36. Monolith K $115 \mathrm{~cm}$}

$3860 \pm 90$

Mor deposit containing charcoal, at rise in Corylus and Calluna pollen values.

$$
\delta^{13} C=-27.5 \%
$$

CAR-37. Monolith K $123 \mathrm{~cm}$

$$
4800 \pm 90
$$

Mor deposit containing charcoal from near elm decline.

\section{CAR-38. Monolith K $126 \mathrm{~cm}$}

$$
\begin{array}{r}
\mathbf{5 4 5 0} \pm \mathbf{7 0} \\
\delta^{13} C=-27.7 \% 0
\end{array}
$$

Mor deposit at mineral interface with high Corylus pollen value.

\section{CAR-15. Monolith L 9cm}

Ombrogenous peat at rising Calluna pollen value.

\section{CAR-16. Monolith L $19 \mathrm{~cm}$}

Ombrogenous peat at rising Gramineae, Plantago, and Potentilla pollen values.

$$
\begin{array}{r}
2340 \pm 70 \\
\delta^{13} C=-27.6 \% 0 \\
\text { and Potentilla }
\end{array}
$$

$$
\begin{array}{r}
1150 \pm 50 \\
\delta^{13} C=-28.2 \% 0
\end{array}
$$


CAR-17. Monolith L $57 \mathrm{~cm}$

$\mathbf{4 7 2 0} \pm \mathbf{8 0}$
$\delta^{13} C=-28.7 \% 0$

Ombrogenous peat at elm decline.

CAR-18. Monolith L $60 \mathrm{~cm}$

$\mathbf{5 8 3 0} \pm \mathbf{7 0}$

$\delta^{13} C=-27.7 \%$

Mor deposit with charcoal at decline in Corylus and increase in Calluna pollen values.

CAR-63. Monolith L 62cm

$4120 \pm 70$

$\delta^{13} C=-27.6 \%$ values.

Ombrogenous peat at decline in Corylus and increase in Calluna pollen

\section{CAR-10. Monolith M $34 \mathrm{~cm}$}

$3330 \pm 60$

Ombrogenous peat at increase in Gramineae and decline in Calluna pollen values.

CAR-11. Monolith M 54cm

$4250 \pm 70$

Ombrogenous peat at increase in Calluna pollen value.

CAR-12. Monolith M $65 \mathrm{~cm}$

$4770 \pm 70$

$\delta^{13} C=-28.1 \%$

Sample from junction of greasy black amorphous peat and ombrogenous peat at decline in Ulmus pollen value.

CAR-13. Monolith $\mathrm{M} 71 \mathrm{~cm}$

$\mathbf{5 7 1 0} \pm \mathbf{8 0}$

Mor deposit at high Calluna pollen value.

$\delta^{13} C=-28.3 \%$

CAR-14. Monolith M $76 \mathrm{~cm}$

$\mathbf{5 7 4 0} \pm \mathbf{8 0}$

Sample of mor deposit at junction with mineral soil, at reduction in Corylus pollen and rise in Calluna pollen values. Comment: $\delta^{13} \mathrm{C}$ value not determined; estimated value of $-28 \% \pm 1$ used in date calculation.

CAR-64. Monolith N $11 \mathrm{~cm}$

$710 \pm 60$

Ombrogenous peat.

$$
\delta^{13} C=-27.6 \%
$$

CAR-83. Monolith N $13 \mathrm{~cm}$

$1230 \pm 60$

Ombrogenous peat with high Calluna and Gramineae pollen values.

CAR-84. Monolith N $26 \mathrm{~cm}$

$4860 \pm 80$

$\delta^{13} \mathrm{C}=-28.8 \%$ ues.

Humified ombrogenous peat with high Corylus and Quercus pollen val- 
CAR-85. Monolith N 32cm

Humified ombrogenous peat.

CAR-66. Monolith N $40 \mathrm{~cm}$

Humified ombrogenous peat.
$4930 \pm 60$

$\delta^{13} C=-28.3 \%$

$4830 \pm 60$

$\delta^{13} C=-28.1 \%$

\section{Waun-Fignen-Felen series}

Peat samples are from series of 15 monoliths and 1 core taken from upland basin and surrounding area at Waun-Fignen-Felen, $5 \mathrm{~km} \mathrm{~N}$ of Abercraf, Powys $\left(51^{\circ} 50^{\prime} \mathrm{N}, 3^{\circ} 42^{\prime} \mathrm{W}\right.$, Natl Grid Ref SS 825179). Coll 1980 by A G Smith and E W Cloutman, Dept Plant Sci, Univ Coll, Cardiff. Site consists of peat-filled late glacial lake basin surrounded by ombrogenous peats, some of which began to form in Mesolithic times. Core WFF/G00, $500 \mathrm{~cm}$ long, came from center of basin and consisted of reedswamp peat and ombrogenous peat. Monolith WFF/E13N, $250 \mathrm{~cm}$ long, from NE edge of basin, comprised basal reedswamp peat overlain by ombrogenous peat. Monolith WFF $/ \mathrm{E} 1 \mathrm{~N}, 60 \mathrm{~cm}$ long, from NE edge of basin, comprised lake muds overlain by ombrogenous peat. Monolith WFF $/ \mathrm{B} 125 \mathrm{~N}, 50 \mathrm{~cm}$ long, from near basin, comprised basal till, $6 \mathrm{~cm}$ mor with ombrogenous peat above. Monolith WFF/B90S, $28 \mathrm{~cm}$ long, from near basin, comprised ironpan podsol, $4 \mathrm{~cm}$ mor with ombrogenous peat above. Monolith WFF/B32S, $55 \mathrm{~cm}$ long, from near basin, comprised basal till, $4 \mathrm{~cm}$ mor with ombrogenous peat above. Monolith WFF/A16W, $60 \mathrm{~cm}$ long, from near basin, comprised basal till with $52 \mathrm{~cm}$ ombrogenous peat above. Monolith WFF/ E188S, $145 \mathrm{~cm}$ long, from near basin, comprised basal till, narrow horizon of humified peat overlain by ombrogenous peat. Monolith WFF $/ \mathrm{A} 0,25 \mathrm{~cm}$ long, from near basin, comprised basal till, $22 \mathrm{~cm}$ black humified peat with mineral particles and included worked Mesolithic flint. Monolith WFF/ A78E, $90 \mathrm{~cm}$ long, from near basin, contained basal till, $8 \mathrm{~cm}$ humified peat with ombrogenous peat above. Monolith WFF $/ B 46.5 \mathrm{~N}, 190 \mathrm{~cm}$ long, from near basin, contained basal till, $48 \mathrm{~cm}$ moderately humified peat and ombrogenous peat. Monolith WFF/F117S, $100 \mathrm{~cm}$ long, from near basin, contained basal till, $8 \mathrm{~cm}$ mor and ombrogenous peat. Monolith WFF/ $\mathrm{EE} 17 \mathrm{E}, 76 \mathrm{~cm}$ long, from near basin, contained basal till, $4 \mathrm{~cm}$ humified peat with ombrogenous peat above. Monolith WFF/NE, $50 \mathrm{~cm}$ long from slope $\mathrm{NE}$ of basin at alt $660 \mathrm{~m}$, contained basal till, $4 \mathrm{~cm}$ humified peat with ombrogenous peat above. Monolith WFF/NP, $50 \mathrm{~cm}$ long, from plateau N of basin at alt $500 \mathrm{~m}$, contained basal till, $5 \mathrm{~cm}$ humified peat with ombrogenous peat above. Monolith WFF/SW, $50 \mathrm{~cm}$ long, from slope to $\mathrm{SW}$ of basin at alt $530 \mathrm{~m}$, contained basal till, thin layer of humifed peat with $48 \mathrm{~cm}$ ombrogenous peat above. Samples taken to construct 3-dimensional vegetational history of area using relative and absolute pollen diagrams (Cloutman, 1983). Monoliths and cores from basin contained aquatic deposits. Monoliths from near basin sites are within $150 \mathrm{~m}$ of aquatic deposits and alts are taken from peat base. All near-basin sites lie between $477 \mathrm{~m}$ and $484 \mathrm{~m}$ alt. All depths refer to tops of monoliths, which are not necessarily at original bog surface. 
CAR-625. WFF/NE $48 \mathrm{~cm}$

$5940 \pm 90$

Black amorphous peat immediately above basal mineral.

CAR-626. WFF/SW $46 \mathrm{~cm}$

$4250 \pm 80$

Ombrogenous peat with Eriophorum.

$\delta^{13} C=-28.4 \%$

CAR-627. WFF/SW $48 \mathrm{~cm}$

$4370 \pm 80$

Black humified peat with charcoal above basal mineral.

CAR-628. WFF/NP $34+36 \mathrm{~cm}$

$\mathbf{5 1 0 0} \pm \mathbf{8 0}$

Ombrogenous peat directly above basal mineral.

$$
\delta^{13} C=-28.5 \%
$$

CAR-421. WFF/E1N $2+3 \mathrm{~cm}$

$4160 \pm 80$

$\delta^{13} \mathrm{C}=-27.7 \%$

Ombrogenous peat with Calluna remains; Calluna pollen percentage increasing sharply.

CAR-422. WFF/E1N $13+14 \mathrm{~cm}$

$4270 \pm 70$
$\delta^{13} C=-27.9 \%$

Coarse Eriophorum peat.

CAR-423. WFF/E1N $21+22 \mathrm{~cm}$

Coarse Eriophorum peat.

CAR-424. WFF/E1N $26+27 \mathrm{~cm}$

$\delta^{13} C=-27.9 \%$

Coarse Eriophorum peat at start of rise of Plantago pollen and fall in Ulmus pollen curve.

CAR-425. WFF/E1N $33+34 \mathrm{~cm}$

$4660 \pm 80$

$\delta^{13} C=-28.3 \%$

Coarse detritus mud with Phragmites rhizomes and Betula and Salix wood fragments.

CAR-426. WFF/E1N $40+41 \mathrm{~cm}$

$$
\begin{array}{r}
\mathbf{4 8 2 0} \pm \mathbf{8 0} \\
\delta^{13} C=-28.7 \%
\end{array}
$$

Coarse detritus mud with Phragmites rhizomes and Sphagnum leaves.

CAR-427. WFF/E1N $42+43 \mathrm{~cm}$

$4900 \pm 80$ $\delta^{13} C=-28.6 \%$

Peat at transition to organic mud, at fall in Ulmus and Corylus pollen and rise in Gramineae pollen levels. 
CAR-428. WFF/E1N $45+46 \mathrm{~cm}$

Organic mud with Juncus seeds.

CAR-429. WFF $/$ E1N $51+52 \mathrm{~cm}$

Organic mud with Juncus seeds.

CAR-430. WFF $/$ E1N $56+57 \mathrm{~cm}$

Organic mud with Juncus seeds.

CAR-635. WFF/E13N 15-18cm

Eriophorum peat.

CAR-634. WFF/E13N $79+80 \mathrm{~cm}$

Eriophorum peat at rise in Plantago pollen curve.

CAR-636. WFF/E13N $143+144 \mathrm{~cm}$

Eriophorum peat.

CAR-637. WFF/E13N $175+176 \mathrm{~cm}$

Eriophorum peat with Calluna at fall in Ulmus pollen curve.

CAR-638. WFF/E13N $223+224 \mathrm{~cm}$

Coarse detritus mud with Phragmites rhizomes. Ulmus pollen values falling, Plantago and Calluna values rising.

CAR-639. WFF/E13N $225+226 \mathrm{~cm}$

$\mathbf{5 2 3 0} \pm \mathbf{8 0}$
$\delta^{13} C=-28.9 \%$

Coarse detritus mud with Phragmites rhizomes.

CAR-640. WFF/E13N $258 \mathrm{~cm}$

Coarse detritus mud at mineral/peat interface.

CAR-686. WFF/G00 121-124cm

Eriophorum peat with Calluna.

CAR-687. WFF/G00 253-256cm

Ombrogenous peat with Eriophorum at rise in Plantago and fall in Ulmus pollen percentages. 
CAR-688. WFF/G00 285-288cm

$\mathbf{5 7 7 0} \pm \mathbf{8 0}$

Coarse detritus mud with Phragmites rhizomes.

$\delta^{13} C=-27.7 \%$

CAR-689. WFF/G00 301-304cm

$6240 \pm 90$

$\delta^{13} C=-27.5 \%$

Phragmites peat with Betula fragments.

CAR-690. WFF/G00 369-376cm

$7460 \pm 90$

$\delta^{13} C=-27.3 \%$

Phragmites peat. Alnus pollen value starts to increase.

CAR-691. WFF/G00 473-476cm

$9340 \pm 110$

$\delta^{13} C=-26.7 \%$ o

Phragmites peat. Decline in Betula and rise in Corylus pollen curves.

CAR-692. WFF/G00 499-504cm

$10,180 \pm 110$

$\delta^{13} C=-26.6 \%$

Organic mud with mineral particles. Comment (AGS): date confirms stratigraphic evidence of early Flandrian age of this level.

CAR-504. WFF/B125N 4cm

$4380 \pm 80$

Ombrogenous peat.

CAR-505. WFF $/$ B $125 \mathrm{~N} 16 \mathrm{~cm}$

$\mathbf{5 2 2 0} \pm \mathbf{8 0}$

Ombrogenous peat.

$\delta^{13} C=-29.0 \%$

CAR-506. WFF/B125N 32cm

$6060 \pm 80$

Peat at decline in Ulmus pollen values.

$\delta^{13} C=-28.7 \%$

CAR-507. WFF/B125N $44 \mathrm{~cm}$

$7610 \pm 90$

$\delta^{13} C=-28.2 \%$

Peat at junction of greasy black peat and ombrogenous peat.

CAR-508. WFF/B125N $46 \mathrm{~cm}$

$7830 \pm 100$

Black greasy ombrogenous peat containing charcoal and Juncus seeds.

CAR-509. WFF/B125N $49 \mathrm{~cm}$

$7940 \pm 100$

Black greasy ombrogenous peat immediately above mineral soil, at fall in Betula and rise in Corylus pollen values.

CAR-499. WFF/B90S 2cm

$4850 \pm 70$

Ombrogenous peat.

$\mathbf{4 8 5 0} \pm \mathbf{7 0}$
$\delta^{13} C=-27.9 \%$ 
CAR-500. WFF/B90S 8cm

Ombrogenous peat at decline in Ulmus pollen value.

CAR-501. WFF/B90S $15 \mathrm{~cm}$ $5490 \pm 80$

$\delta^{13} C=-28.3 \%$

Peat at interface between basal mor and ombrogenous peat, at fall in Calluna pollen value.

CAR-502. WFF/B90S $18+19 \mathrm{~cm}$

$6490 \pm 90$

Organic mineral soil at rising Alnus and high Calluna pollen values.

CAR-337. WFF/B32S $8+9 \mathrm{~cm}$

$4060 \pm 70$

Ombrogenous peat.

CAR-338. WFF/B32S $16+17 \mathrm{~cm}$

$4030 \pm 70$

Ombrogenous peat.

$\delta^{13} C=-28.4 \%$

CAR-339. WFF/B32S $24+\mathbf{2 5} \mathrm{cm}$

$4870 \pm 80$

Ombrogenous peat at fall in Ulmus pollen value.

$\delta^{13} C=-28.2 \%$

CAR-340. WFF/B32S $32+33 \mathrm{~cm}$

$\mathbf{5 2 0 0} \pm \mathbf{8 0}$

Ombrogenous peat.

$\delta^{13} \mathrm{C}=-28.5 \%$

CAR-341. WFF/B32S $40+41 \mathrm{~cm}$

$\mathbf{5 7 7 0} \pm \mathbf{8 0}$

Ombrogenous peat with mineral particles.

$\delta^{13} \mathrm{C}=-28.1 \%$

CAR-342. WFF/B32S $49 \mathrm{~cm}$

$\mathbf{5 8 6 0}+\mathbf{8 0}$

$\delta^{13} C=-28.3 \%$

Peat at interface between black mor and ombrogenous peat, at rising Alnus and high Calluna pollen values.

CAR-343. WFF/B32S $53 \mathrm{~cm}$

$6520+100$

$\delta^{13} C=-27.9 \%$

Mor deposit with much mineral material immediately above mineral soil, at high levels of Corylus and Calluna pollen.

CAR-323. WFF/A16W 8cm

Ombrogenous peat.

$$
\begin{array}{r}
\mathbf{3 8 0 0} \pm \mathbf{7 0} \\
\delta^{13} C=-28.5 \% 0
\end{array}
$$


CAR-324. WFF/A16W $15+16 \mathrm{~cm}$ $4350 \pm 70$

Ombrogenous peat.

CAR-325. WFF/A16W $23+24 \mathrm{~cm}$

Ombrogenous peat.

CAR-326. WFF/A16W $32 \mathrm{~cm}$

$\mathbf{5 2 4 0} \pm \mathbf{8 0}$

Black greasy peat with much charcoal, at fall in Ulmus pollen value.

\section{CAR-327. WFF/A16W 40cm}

Black greasy peat with mineral particles.

CAR-328. WFF/A16W $48 \mathrm{~cm}$

Black greasy peat with mineral particles.

CAR-329. WFF/A16W 52cm

Black greasy peat directly above mineral soil, at high Corylus pollen value.

CAR-61. WFF $/$ A0 $17 \mathrm{~cm}$

$\mathbf{7 7 0 0} \pm 90$

$\delta^{13} C=-28.4 \%$ o

Black humified peat with mineral material at level of worked Mesolithic flint lying $3 \mathrm{~cm}$ above mineral soil.

CAR-330. WFF/A78E $12+13 \mathrm{~cm}$

$\mathbf{2 2 9 0} \pm \mathbf{7 0}$
$\delta^{13} C=-27.7 \% 0$

Ombrogenous peat.

CAR-331. WFF/A78E $28+29 \mathrm{~cm}$

$$
\begin{array}{r}
\mathbf{3 0 0 0} \pm \mathbf{6 0} \\
\delta^{13} C=-27.9 \% \text { o }
\end{array}
$$

Ombrogenous peat at rise in Fraxinus and Narthecium pollen values.

CAR-332. WFF/A78E $44+45 \mathrm{~cm}$

$3370 \pm 70$

Ombrogenous peat.

$$
\delta^{13} C=-28.1 \%
$$

CAR-333. WFF/A78E $52+53 \mathrm{~cm}$

$3710 \pm 60$

$\delta^{13} C=-28.1 \%$

Ombrogenous peat at fall in Ulmus and rise in Plantago pollen values.

CAR-334. WFF $/$ A78E $60+61 \mathrm{~cm}$

$4310 \pm 80$

Ombrogenous peat with some mineral particles.

$$
\delta^{13} C=-28.3 \%
$$


CAR-335. WFF/A78E $77 \mathrm{~cm}$

$5530 \pm 90$

$\delta^{13} \mathrm{C}=-28.0 \%$

Dark brown greasy peat with mineral particles and charred Calluna fragments.

CAR-336. WFF/A78E $81 \mathrm{~cm}$

$\mathbf{5 3 4 0} \pm \mathbf{8 0}$

$\delta^{13} C=-27.4 \%$

Dark brown greasy peat directly above mineral soil, at high values of Corylus and Calluna pollen.

CAR-439. WFF/E188S $2+3 \mathrm{~cm}$

$1630 \pm 70$

$\delta^{13} C=-28.5 \%$

Ombrogenous peat with Eriophorum and Calluna fragments.

CAR-440. WFF/E188S $31+32 \mathrm{~cm}$

$2530 \pm 70$

Ombrogenous peat at rising Fraxinus and Narthecium pollen values.

CAR-441. WFF/E188S $55+56 \mathrm{~cm}$

$3150 \pm 70$

Ombrogenous peat.

$\delta^{13} C=-27.9 \%$

CAR-442. WFF $/$ E188S $83 \mathrm{~cm}$

$3520 \pm 70$

$\delta^{13} C=-28.2 \%$

Ombrogenous peat with rising Plantago and Pteridium and declining Ulmus pollen levels.

CAR-443. WFF/E188S $99+100 \mathrm{~cm}$

$4220 \pm 80$ lation.

Ombrogenous peat. Comment: $\delta^{13} \mathrm{C}=-28 \% 0 \pm 1$ used in date calcu-

CAR-444. WFF/E188S $119+120 \mathrm{~cm}$

$4690 \pm 80$

Ombrogenous peat.

$\delta^{13} C=-28.4 \% 0$

CAR-445. WFF/E188S $144 \mathrm{~cm}$

$\mathbf{6 2 1 0} \pm 90$

$\delta^{13} C=-28.4 \% 0$

Black peat with charcoal and some mineral particles directly above mineral soil at declining Calluna pollen curve.

CAR-406. WFF/B46.5N $7+8 \mathrm{~cm}$

$1150 \pm 60$

$\delta^{13} C=-27.0 \%$

Ombrogenous peat with Calluna and Eriophorum fragments.

CAR-407. WFF/B46.5N $39+40 \mathrm{~cm}$

$2110 \pm 70$

Ombrogenous peat with Calluna and Eriophorum fragments. 
CAR-408. WFF/B46.5N $71+72 \mathrm{~cm}$

CAR-409. WFF/B46.5N $87+88 \mathrm{~cm}$

Ombrogenous peat.

$$
\delta^{13} C=-27.5 \%
$$

Ombrogenous peat at rising Fraxinus and Narthecium pollen curves.

CAR-410. WFF/B46.5N $119+120 \mathrm{~cm}$

$$
\begin{array}{r}
\mathbf{3 0 3 0} \pm \mathbf{7 0} \\
\delta^{13} C=-28.0 \%
\end{array}
$$

Humified ombrogenous peat at rising Plantago and Pteridium and declining Ulmus pollen curves.

\section{CAR-411. WFF/B46.5N $151+152 \mathrm{~cm}$}

$4450 \pm 80$

Ombrogenous peat.

CAR-412. WFF/B46.5N $167+168 \mathrm{~cm}$

$\mathbf{5 1 4 0} \pm \mathbf{8 0}$ lation.

Ombrogenous peat. Comment: $\delta^{13} \mathrm{C}=-27.7 \% 0 \pm 1$ used in date calcu-

CAR-413. WFF/B46.5N $183+184 \mathrm{~cm}$

$5670 \pm 70$

Humified ombrogenous peat with much charcoal directly above peat/ soil interface. Comment: $\delta^{13} \mathrm{C}=-27.7 \% 01$ used in date calculation.

CAR-629. WFF/F117S $48 \mathrm{~cm}$

$3720 \pm 70$

Ombrogenous peat with Calluna and Eriophorum fragments.

CAR-630. WFF/F117S 65cm

Ombrogenous peat.

CAR-631. WFF/F117S $80 \mathrm{~cm}$

Humified ombrogenous peat.

CAR-632. WFF/F117S $83 \mathrm{~cm}$
$4440 \pm 70$

$\delta^{13} C=-28.0 \%$

$\mathbf{5 4 9 0} \pm \mathbf{8 0}$

$\delta^{13} C=-28.8 \%$

$\mathbf{5 7 8 0} \pm \mathbf{8 0}$

$\delta^{13} C=-28.9 \%$

Black humified peat at rising Alnus and falling Pinus and Calluna pollen values.

CAR-633. WFF/F117S $91 \mathrm{~cm}$

$$
6770 \pm 90
$$$$
\delta^{13} C=-28.6 \%
$$

Black humified peat with much mineral material directly above mineral soil interface. 
CAR-624. WFF/EE17.5E $32+33 \mathrm{~cm}$

Ombrogenous peat with Calluna and Eriophorum fragments.

CAR-623. WFF/EE17.5E $51 \mathrm{~cm}$

$\mathbf{4 8 1 0} \pm \mathbf{8 0}$
$\delta^{13} C=-28.5 \%$

Humified ombrogenous peat at decline of Ulmus pollen value.

CAR-622. WFF $/$ EE17.5E $63 \mathrm{~cm}$

Humified ombrogenous peat.

CAR-621. WFF/EE17.5E $69 \mathrm{~cm}$

Black humified ombrogenous peat with mineral particles at declining Pinus pollen value.

General Comment (AGS): dates from basal levels of monoliths from basin margin show variations in initiation of growth of organic deposits of ca $2000 \mathrm{yr}$.

\section{Cefn Gwernffrwd series}

Peat samples are from 3 cores taken on plateau near stone circle and alignment at Cefn Gwernffrwd (Briggs, 1975; Morgan \& Ruggles, 1976). Site, at alt of ca $400 \mathrm{~m}$, is $15 \mathrm{~km} \mathrm{~N}$ of Llandovery, Dyfed $\left(52^{\circ} 08^{\prime} \mathrm{N}, 3^{\circ} 50^{\prime}\right.$ W). Coll and subm 1975 by F M Chambers, Dept Plant Sci, Univ Coll, Cardiff. Core A was taken close to archaeol site (Natl Grid Ref SN 738494), Core B was taken ca 150m NW (Natl Grid Ref SN 735494), and Core C ca $500 \mathrm{~m} \mathrm{SE}$ (Natl Grid Ref SN 743492). Cores A and B were of blanket peat and Core $\mathrm{C}$ was of deeper basin peat. They were taken as part of study of peat initiation in upland S Wales (Chambers, 1980; 1982b; 1983c).

\section{CAR-76. CG Core A $9.5-10 \mathrm{~cm}$}

$$
\text { Modern }
$$
$\delta^{13} \mathrm{C}=-31.2 \%$

Peat at transition from high Calluna to high Gramineae and Cyperaceae pollen values.

CAR-75. CG Core A $23-24 c m$

$$
\delta^{13} C=\begin{array}{r}
\mathbf{7 3 0} \pm \mathbf{4 0} \\
-28.2^{0} \% 0
\end{array}
$$

Peat at change from high Gramineae to high Calluna pollen values.

CAR-74. CG Core A $43-44 \mathrm{~cm}$

$$
\begin{array}{r}
1900 \pm 45 \\
\delta^{13} C=-28.3 \% 0
\end{array}
$$

Peat at Corylus pollen min and increasing Gramineae pollen values.

CAR-73. CG Core A $49-50 \mathrm{~cm}$

$$
\begin{array}{r}
2030 \pm \mathbf{4 5} \\
\delta^{13} C=-28.4 \% 0
\end{array}
$$

Peat at decline in Betula and Gramineae and increase in Corylus, Plantago, and Cyperaceae pollen values. 
CAR-39. CG Core A $58-59 \mathrm{~cm}$ $2730 \pm 70$

Peat at final episode of high level of Plantago pollen.

$$
\delta^{13} C=-28.4 \%
$$

CAR-71. CG Core A $62-63 \mathrm{~cm}$

$\mathbf{2 8 6 0} \pm \mathbf{5 0}$

Peat at start of rise in level of Plantago pollen.

$$
\delta^{13} \mathrm{C}=-28.7 \%
$$

CAR-19. CG Core A 68-69cm

$3470 \pm 70$

Basal layer of peat with charcoal in horizon.

$$
\delta^{13} \mathrm{C}=-28.2 \%
$$

CAR-44. CG Core B $26-27 \mathrm{~cm}$

$380 \pm 60$

$\delta^{13} C=-28.5 \%$

Peat at change from high to lower Calluna pollen value.

CAR-43. CG Core B 51-52cm

$2140 \pm 70$

$\delta^{13} C=-28.3 \%$

Peat marking rise in Calluna and Cyperaceae pollen values.

\section{CAR-42. CG Core B 64-65cm}

Peat at resurgence of Plantago pollen.

CAR-7. CG Core B 72-73cm
$3720 \pm 70$

$\delta^{13} C=-28.5 \%$

$3850 \pm 80$

$\delta^{13} C=-28.7 \%$

Peat at decline in Ulmus pollen, close to first appearance of Plantago pollen at $75 \mathrm{~cm}$.

CAR-41. CG Core B 89-90cm

$4990 \pm 80$

Peat at start of sustained rise in Alnus pollen value.

CAR-40. CG Core B $104-105 \mathrm{~cm}$

$5490 \pm 80$

Basal layer of peat.

CAR-93. CG Core C $114-118 \mathrm{~cm}$

$2580 \pm 60$

Peat at peak in Calluna pollen value.

CAR-20. CG Core C $170-174 \mathrm{~cm}$

$\mathbf{3 3 4 0} \pm \mathbf{8 0}$

$\delta^{13} C=-28.6^{6} \%$

Peat at decline in Calluna and increasing Gramineae pollen values.

CAR-45. CG Core C $212-216 \mathrm{~cm}$

$4930 \pm 90$

Peat at Ulmus pollen decline. 
CAR-94. CG Core C $236-240 \mathrm{~cm}$

Peat at peak in Ulmus pollen and declining Corylus pollen values.

CAR-95. CG Core C $258-262 \mathrm{~cm}$

$\mathbf{5 8 7 0} \pm \mathbf{8 0}$

$\delta^{13} C=-27.8 \%$

Peat with abundant Sphagnum spores and marking increase in Calluna pollen value.

CAR-96. CG Core C 304-308cm

$6820 \pm 90$

Peat at start of rise in Alnus pollen value.

$\delta^{13} C=-28.4 \%$

CAR-97. CG Core C $412-420 \mathrm{~cm}$

$9430 \pm 110$

Coarse reedswamp peat.

CAR-98. CG Core C $446-452 \mathrm{~cm}$

$\delta^{13} C=-28.2 \%$

Coarse reedswamp peat at start of rise in Corylus pollen.

CAR-99. CG Core C 478-482cm

$9070 \pm 120$

$\delta^{13} C=-26.6 \%$

Fine detritus mud overlying clayey mud at $484 \mathrm{~cm}$.

\section{Cefn Fford series}

Peat samples are from core taken by F M Chambers, from shallow blanket peat at alt $600 \mathrm{~m}$. Sampling point lies $95 \mathrm{~m} \mathrm{~W}$ of triangulation point at Cefn Fford, $23 \mathrm{~km} \mathrm{~N}$ of Bridgend, Mid Glamorgan $\left(51^{\circ} 43^{\prime} \mathrm{N}, 3^{\circ} 35^{\prime} \mathrm{W}\right.$, Natl Grid Ref SN 906032). Coll and subm 1974 by FMC. Comment (FMC): site overlies Pennant sandstone. Samples taken in conjunction with investigations of blanket peat initiation in S Wales (Chambers, 1981; 1982a). All samples are fine fractions of peat pretreated by FMC. Depths refer to modern surface.

CAR-47. CFF 4-5cm

$\mathbf{2 9 7 0} \pm \mathbf{5 0}$

Peat at rise in Gramineae pollen concentration. Comment: much older than expected, probably due to presence of dead carbon deposited as soot during industrial revolution (Chambers, Dresser, \& Smith, 1979).

\section{CAR-106. CFF 8-10cm}

CAR-46. CFF 14-15cm

CAR-107. CFF 16-18cm

$$
\begin{array}{r}
\mathbf{9 3 0} \pm \mathbf{5 0} \\
\delta^{13} C=-28.2 \% 0 \\
\mathbf{1 4 1 0} \pm \mathbf{6 0} \\
\delta^{13} C=-27.7 \% 0 \\
\mathbf{1 6 6 0} \pm \mathbf{6 0} \\
\delta^{13} C=-28.5 \% 0
\end{array}
$$


CAR-8. CFF 22-24cm

Peat with high value of Ericaceae pollen.

\section{CAR-9. CFF 24-26cm}

$3160 \pm 60$

$\delta^{13} C=-27.9 \%$

$3630 \pm 80$

$\delta^{13} \mathrm{C}=-28.2 \%$

Peat from mineral/peat interface.

\section{Coed Taf series}

Peat samples are from 3 cores taken within $200 \mathrm{~m}$ of each other from plateau overlying gray grits and S of area of carboniferous limestone $7 \mathrm{~km}$ NW of Merthyr Tydfil, Mid Glamorgan $\left(51^{\circ} 47^{\prime} \mathrm{N}, 3^{\circ} 28^{\prime} \mathrm{W}\right)$. Coll and subm 1975 by F M Chambers. Comment (FMC): Core A comprised amorphous blanket peat at alt 400m (Natl Grid Ref SN 988108). Core B was of amorphous blanket peat twice depth of Core A, $51 \mathrm{~m}$ to E (Natl Grid Ref SN 989108 ), at alt $400 \mathrm{~m}$. Core $\mathrm{C}$ was taken from deepest part of basin peat area ca $200 \mathrm{~m}$ S of blanket peat sites at alt $380 \mathrm{~m}$ (Natl Grid Ref SN 988107). Samples taken as part of study of blanket peat initiation in S Wales uplands; Core $\mathrm{C}$ was coll for inf on pre-blanket peat vegetation at site (Chambers, $1980 ; 1983 b)$.

\section{CAR-82. CT Core A 6-8cm}

$$
\begin{array}{r}
\mathbf{3 7 1 0} \pm \mathbf{9 0} \\
\delta^{13} C=-26.5 \% \text {. }
\end{array}
$$

Peat antedating substantial rise in Gramineae pollen representation. Comment: much older than expected, probably due to presence of inactive carbon in soot deposited in industrial revolution, as in CAR-47. Humic acid fraction also dated; CAR-82C, $70 \pm 55$. Result indicates inactive carbon deposition rate of order of $4 \mathrm{~g} / \mathrm{m}^{2} / \mathrm{yr}$ over estimated $32 \mathrm{yr}$ accumulation period of sample (Chambers, Dresser, \& Smith, 1979).

\section{CAR-81. CT Core A 11-13cm}

$140 \pm 50$

Peat at final decline of Corylus pollen.

$$
\delta^{13} C=-27.9 \%
$$

CAR-80. CT Core A 14-16cm

Peat at change from Gramineae to Calluna pollen types.

CAR-79. CT Core A $20-22 \mathrm{~cm}$

$350 \pm 70$

$$
\delta^{13} \mathrm{C}=-28.0^{0} \%
$$
pollen.

Peat containing abundant Campanulaceae, Cyperaceae, and Potentilla

CAR-78. CT Core A $27-28 \mathrm{~cm}$

$$
\mathbf{1 2 0 0} \pm \mathbf{5 0}
$$

Well-humified peat at start of decline in Calluna pollen. 
CAR-77. CT Core A $28-30 \mathrm{~cm}$

$1440 \pm 60$

$\delta^{13} C=-28.6 \%$ pollen.

Peat from mineral/peat transition containing abundant Calluna

CAR-50. CT Core B $68-70 \mathrm{~cm}$

$1540 \pm 60$

$\delta^{13} C=-28.2 \%$ peak.

Humic acid fraction of peat at point where Cyperaceae pollen is at

CAR-48. CT Core B 70-72cm

$1310 \pm 70$
$\delta^{13} C=-28.0^{\circ} \% 0$

Peat at mineral/peat interface. Comment: humic acid fraction also dated; CAR-48C, $1630 \pm 60$.

CAR-92. CT Core C $140-146 \mathrm{~cm}$

$\mathbf{2 9 9 0} \pm \mathbf{5 0}$
$\delta^{13} C=-27.6^{0} \% 0$

Calluna Eriophorum peat at min in arboreal pollen.

CAR-91. CT Core C 290-294cm

$4620 \pm 70$
$\delta^{13} C=-28.1 \% 0$

Sphagnum Cyperaceae peat at decline in Ulmus pollen.

CAR-90. CT Core C $326-330 \mathrm{~cm}$

Fen peat with wood fragments at decline in Corylus pollen.

CAR-89. CT Core C 342-346cm

$6650 \pm 90$

$\delta^{13} C=-28.4 \%$

Peat at rise in Alnus and decline in Pinus pollen. Boreal-Atlantic transition sensu Mitchell (1956).

CAR-88. CT Core C 350-353cm

$6890 \pm 110$

Basal layer of reed peat directly above mineral.

\section{Brecon Beacons series}

Peat samples are from monolith taken above and $\mathrm{S}$ of $\mathrm{N}$ facing Old Red Sandstone scarp, $3 \mathrm{~km}$ SE of peak of Pen-y-Fan Mt, Powys $\left(51^{\circ} 52^{\prime} \mathrm{N}\right.$, $3^{\circ} 23^{\prime}$ W, Natl Grid Ref SO 043196 ) at alt $715 \mathrm{~m}$. Coll and subm 1975 by F M Chambers. Comment (FMC): site is at highest elev in study of blanket peat initiation in S Wales uplands (Chambers, 1980; 1981; 1982a).

CAR-59. 19-21 cm $470 \pm 70$ pollen.

Fibrous blanket peat at rise in Calluna and start of rise in Empetrum 
CAR-58. $38-40 \mathrm{~cm}$

$1860 \pm 80$

$$
\delta^{13} C=-28.2^{0} \%
$$

Fibrous blanket peat at fall in Cyperaceae and rise in Calluna pollen.

CAR-57. $49-51 \mathrm{~cm}$

$2070 \pm 70$

Peat at peak in Cyperaceae pollen.

$$
\delta^{13} C=-27.6 \%
$$

CAR-56. $63-65 \mathrm{~cm}$

$2340 \pm 70$

Blanket peat.

$$
\delta^{13} C=-27.6 \%
$$

CAR-55. $67-71 \mathrm{~cm}$ $\mathbf{2 8 7 0} \pm \mathbf{8 0}$

Blanket peat.

$\delta^{13} C=-27.9 \%$

CAR-54. $\quad 75-77 \mathrm{~cm}$

$\mathbf{2 8 8 0} \pm \mathbf{8 0}$
$\delta^{13} C=-28.2^{\circ} \%$

Peat at fall in Calluna and rise in Gramineae, Cyperaceae, and start of rise in Fraxinus pollen.

CAR-53. $\quad 81-83 \mathrm{~cm}$

$3430 \pm 70$

Peat postdating fall in Corylus and rise in Calluna pollen.

CAR-52. $\quad 95-97 \mathrm{~cm}$

$4090 \pm 70$

$\delta^{13} C=-28.6 \%$

Blanket peat at fall in Calluna and rise in Cyperaceae pollen.

CAR-51. $100-102 \mathrm{~cm}$

$$
\begin{array}{r}
4380 \pm 70 \\
\delta^{13} C=-28.7 \% 0
\end{array}
$$
pollen.

Basal blanket peat at mineral peat transition, marking drop in Corylus

\section{Llangorse Lake series}

Samples of organic nekron mud sub-sampled from core taken using pneumatic Mackereth corer from center of Llangorse Lake, Powys $\left(51^{\circ} 55^{\prime}\right.$ N, $3^{\circ} 16^{\prime}$ W, Natl Grid Ref SO 135265). Coll and subm 1975 by R Jones, Dept Plant Sci, Univ Coll, Cardiff. Samples taken in conjunction with studies in diatom, pollen, and chemical content of core. Previous cores taken in $\mathrm{E}$ basin of lake (Jones et al, 1978). Comment: acid wash pretreatment.

\section{CAR-87. 280-290cm}

$$
\begin{array}{r}
\mathbf{6 1 0} \pm \mathbf{7 0} \\
\delta^{13} C=-30.5 \% 0
\end{array}
$$
clay.

Organic nekron mud from directly below transition to red-brown silty 
CAR-86. $300-310 \mathrm{~cm}$

Organic nekron mud.

General Comment: CAR-86 agrees with results obtained previously (SSR-129 to -132: R, 1974, v 16, p 247-248). CAR-87 is far too young.

\section{England}

\section{Foulshaw Moss series}

Peat samples are from Foulshaw Moss, $10 \mathrm{~km}$ SW of Kendal, Cumbria $\left(54^{\circ} 15^{\prime} \mathrm{N}, 2^{\circ} 50^{\prime} \mathrm{W}\right.$, Natl Grid Ref SD 458837). Samples coll directly from exposed peat face and as sub-samples from 2 monoliths of peat which were taken from peat face for strat and palynol study. Coll 1981 by G T Wimble and A G Smith, Dept Plant Sci, Univ Coll, Cardiff, and subm 1981 by GTW. Monolith FM1/A was taken at depth $50 \mathrm{~cm}$ below present bog surface. Monolith FM1/B was taken at depth $25 \mathrm{~cm}$. Depths for all samples are relative to present bog surface.

CAR-545. FM1/A 63-64cm $1450 \pm 70$

Unhumified Sphagnum peat dating end of Betula pollen peak.

CAR-546. FM1/A 73-74cm

$1680 \pm 60$

Moderately humified Sphagnum peat dating top of retardation layer.

CAR-547. FM1/A 79-80cm

$1690 \pm 60$

Moderately humified Sphagnum peat dating max clearance of forest as indicated by high NAP (non-arboreal pollen) values.

CAR-548. FM1/A 89-90cm

$2020 \pm 60$

$\delta^{13} \mathrm{C}=-27.4 \%$ o

Well humified Sphagnum peat dating start of cereal pollen curve in clearance episode.

CAR-549. FM1/A 93-94cm

$\mathbf{2 0 9 0} \pm \mathbf{7 0}$
$\delta^{13} C=-27.0 \%$

Well humified Sphagnum peat dating start of clearance episode.

CAR-550. FM1/A 104-105cm

$2280 \pm 70$

CAR-550. FM1/A 104-105cm layer.

$\delta^{13} C=-27.2 \%$ 
CAR-551. FM1/A 110-111 cm

Unhumified Sphagnum peat dating start of Sphagnum imbricatum infill of pool.

CAR-552. FM1/A 117-118cm

$3060 \pm 70$

Sphagnum cuspidatum peat dating base of recurrence surface pool peat.

CAR-553. FM1/A 118-119cm

$3140 \pm 70$

Well humified Sphagnum peat dating top of retardation layer and start of small clearance episode.

CAR-554. FM1/A 137-138cm

$3690 \pm 70$

$\delta^{13} C=-27.2 \%$

Well humified Sphagnum peat dating fall in Ulmus pollen percentages and rise in NAP percentages.

CAR-536. FM1/B 42-43cm

$1820 \pm 70$

$\delta^{13} C=-27.1 \%$

Slightly humified Sphagnum peat with some Eriophorum vaginatum dating end of clearance episode.

\section{CAR-537. FM1/B 48-49cm}

$1840 \pm 70$

Well humified Sphagnum peat dating max clearance of forest as indicated by high NAP percentages.

\section{CAR-538. FM1/B 54-55cm}

$2200 \pm 70$

$\delta^{13} C=-27.3 \%$

Well humified Sphagnum peat dating start of cereal pollen curve in clearance episode.

CAR-539. FM1/B 62-63cm

$2420 \pm 70$

$\delta^{13} C=-27.2^{\%} \%$ est.

Well humified Sphagnum peat dating start of gradual clearance of for-

CAR-540. FM1/B 80-81 cm

$$
\begin{array}{r}
\mathbf{2 7 4 0} \pm \mathbf{7 0} \\
\delta^{13} C=-26.1 \% 0
\end{array}
$$
phase.

Well humified Sphagnum peat with some Calluna dating end of pollen

CAR-541. FM1/B 89-90cm

$$
\begin{array}{r}
\mathbf{2 9 2 0} \pm \mathbf{7 0} \\
\delta^{13} C=-26.5 \% \text {. }
\end{array}
$$

Moderately humified Sphagnum peat dating end of clearance episode. 
CAR-542. FM1/B 95-96cm

$3110 \pm 70$

$\delta^{13} C=-27.4 \%$ o sode.

Moderately humified Sphagnum peat dating start of clearance epi-

CAR-543. FM1/B 125-126cm

$3590 \pm 70$

Well humified Sphagnum peat dating fall in Ulmus pollen percentages and rise in NAP percentages.

CAR-544. FM1/B 143-144cm

$$
\begin{array}{r}
\mathbf{3 8 7 0} \pm \mathbf{7 0} \\
\delta^{13} C=-26.7 \% 0
\end{array}
$$

Well humified Sphagnum peat dating small clearance episode and fall in Ulmus pollen percentages.

CAR-577. FM2 $70 \mathrm{~cm}$

$$
1320 \pm 60
$$

Eriophorum vaginatum tussock dating top of retardation layer.

CAR-578. FM3 50cm

$1370 \pm 70$

Eriophorum vaginatum and some Calluna dating top of retardation layer.

CAR-579. FM4 $35 \mathrm{~cm}$

$$
\begin{array}{r}
1500 \pm 60 \\
\delta^{13} C=-26.8 \%
\end{array}
$$

Well humified Sphagnum peat dating top of retardation layer.

CAR-580. FM5 $35 \mathrm{~cm}$

$1470 \pm 60$

$\delta^{13} C=-27.2 \%$ layer.

Well humified Calluna and Sphagnum peat dating top of retardation

CAR-581. FM6 $75 \mathrm{~cm}$

$$
\begin{array}{r}
\mathbf{2 1 6 0} \pm \mathbf{7 0} \\
\delta^{13} C=-31.0 \%
\end{array}
$$

Sphagnum cuspidatum mud dating small lens of pool peat.

CAR-582. FM8 $115 \mathrm{~cm}$

Eriophorum vaginatum tussock dating top of retardation layer.

CAR-583. FM9 $120 \mathrm{~cm}$

$$
\begin{array}{r}
3200 \pm 70 \\
\delta^{13} C=-27.0 \%
\end{array}
$$

Well humified Sphagnum peat dating top of retardation layer.

CAR-584. FM10 $105 \mathrm{~cm}$

$$
\begin{array}{r}
3180 \pm 70 \\
\delta^{13} C=-26.9 \%
\end{array}
$$

Eriophorum vaginatum peat dating top of retardation layer. 
CAR-585. FM11 $95 \mathrm{~cm}$

Calluna and Sphagnum peat dating top of retardation layer.

CAR-586. FM12 85cm

$2840 \pm 70$

Calluna and Sphagnum peat dating top of retardation layer.

CAR-587. FM13 $105 \mathrm{~cm}$

$$
3200 \pm 70
$$

Calluna and Sphagnum peat dating top of retardation layer.

CAR-590. FM22 $115 \mathrm{~cm}$

$$
\begin{array}{r}
3160 \pm 70 \\
\delta^{13} C=-26.8 \%
\end{array}
$$

Well humified Sphagnum and Eriophorum peat dating top of retardation layer.

\section{Helsington Moss series}

Peat samples are from cutting on Helsington Moss, $5 \mathrm{~km}$ SW of Kendal, Cumbria (54 $17^{\circ} \mathrm{N}, 2^{\circ} 49^{\prime} \mathrm{W}$, Natl Grid Ref SD 466889). Coll 1982 by G T Wimble and L A Morgan, Dept Plant Sci, Univ Coll, Cardiff, and subm by GTW. Samples were taken from 4 monoliths of peat and 5 discrete samples which were coll at intervals along exposed peat face for strat and palynol studies. Monolith HM1 was taken at depth $115 \mathrm{~cm}, \mathrm{HM} 2$ at $110 \mathrm{~cm}, \mathrm{HM} 3$ at $110 \mathrm{~cm}$, and HM4 at $40 \mathrm{~cm}$. All depths are relative to present mire surface.

CAR-594. HM1 116-118cm

$$
\begin{array}{r}
\mathbf{2 7 7 0} \pm \mathbf{7 0} \\
\delta^{13} C=-26.9 \% 0
\end{array}
$$

Unhumified Sphagnum peat dating base of recurrence surface.

CAR-595. HM1 118-119cm

$$
2790 \pm 70
$$$$
\delta^{13} C=-27.2 \%
$$

Well humified Sphagnum peat dating top of retardation layer.

CAR-596. HM1 $127-128 \mathrm{~cm}$

$3230 \pm 70$

Well humified Sphagnum peat dating base of retardation layer and max forest clearance as indicated by high NAP values.

CAR-597. HM1 135-136cm

$3320 \pm 70$

Well humified Sphagnum peat dating top of retardation layer.

CAR-598. HM2 114-116cm

$2810 \pm 70$

$\delta^{13} C=-26.5 \%$

Unhumified Sphagnum peat dating base of recurrence surface. 
CAR-599. HM2 116-117cm

Fairly humified Sphagnum peat dating top of retardation layer.

CAR-602. HM2 122-124cm

$2990 \pm 70$

$\delta^{13} C=-27.1 \% 0$

Fairly humified Sphagnum peat dating base of retardation layer and max forest clearance as indicated by high NAP values.

CAR-603. HM2 137-139cm

$$
\begin{array}{r}
3200 \pm 70 \\
\delta^{13} C=-27.1 \% 0
\end{array}
$$

Slightly humified Sphagnum peat dating slight increase in peat humification and start of clearance episode.

CAR-604. HM2 154-155cm

$$
\begin{array}{r}
\mathbf{3 6 6 0} \pm \mathbf{7 0} \\
\delta^{13} C=-27.3 \% 0
\end{array}
$$

Well humified Sphagnum peat dating top of retardation layer.

CAR-605. HM3 $133 \mathrm{~cm}$

$$
\begin{array}{r}
3390 \pm \mathbf{7 0} \\
\delta^{13} C=-26.1 \% 0
\end{array}
$$

Top of Eriophorum vaginatum tussock dating top of retardation layer.

CAR-609. HM4 46-47cm

$$
\begin{array}{r}
1860 \pm 60 \\
\delta^{13} C=-26.7 \% 0
\end{array}
$$

Well humified Sphagnum peat dating top of retardation layer.

CAR-610. HM4 50-51 cm

$1970 \pm 70$

Well humified Sphagnum peat dating base of retardation layer.

CAR-611. HM4 56-57cm

$2190 \pm 70$

Unhumified Sphagnum peat dating base of recurrence surface and start of clearance episode.

CAR-612. HM4 57-58cm

$$
\begin{array}{r}
\mathbf{2 2 4 0} \pm \mathbf{7 0} \\
\delta^{13} C=-27.3 \% 0
\end{array}
$$

Well humified Sphagnum peat dating top of retardation layer and start of clearance episode.

CAR-613. HM4 66-67cm

$$
\begin{array}{r}
\mathbf{2 4 8 0} \pm \mathbf{7 0} \\
\delta^{13} C=-26.5 \% 0
\end{array}
$$

Well humified Sphagnum and Eriophorum peat dating base of retardation layer.

CAR-614. HM4 72-73cm

$2470 \pm 70$

Well humified Sphagnum peat dating humified layer.

$$
\delta^{13} C=-27.2 \%
$$


CAR-615. HM4 81-82cm

$$
2600 \pm 70
$$

Well humified Sphagnum peat dating top of humified layer.

CAR-606. HM11 $155 \mathrm{~cm}$

$3620 \pm 70$

Well humified Sphagnum peat dating top of retardation layer.

CAR-607. HM12 $125 \mathrm{~cm}$

Sphagnum peat dating top of retardation layer.

CAR-608. HM13 $130 \mathrm{~cm}$

Well humified Sphagnum and Eriophorum peat dating retardation layer.

CAR-616. HM27 95cm

Top of Eriophorum tussock below monolith HM4 dating top of retardation layer.

CAR-617. HM28 70cm

$$
\begin{array}{r}
2150 \pm 70 \\
\delta^{13} C=-26.6 \% 0
\end{array}
$$

Well humified Sphagnum peat dating retardation layer.

\section{CAR-683. HM1 40-43cm}

$$
\begin{array}{r}
\mathbf{3 6 7 0} \pm \mathbf{7 0} \\
\delta^{13} C=-27.4 \% 0
\end{array}
$$

Well humified Sphagnum peat with some Eriophorum and Calluna dating end of Betula pollen peak.

CAR-684. HM3 51-52cm

$$
\begin{array}{r}
3550 \pm 70 \\
\delta^{13} C=-27.3 \% 0
\end{array}
$$

Well humified Sphagnum peat with some Eriophorum dating end of Betula pollen peak.

\section{CAR-685. HM3 16-18cm}

$$
\begin{array}{r}
\mathbf{2 7 5 0} \pm \mathbf{7 0} \\
\delta^{13} C=-26.1 \% 0
\end{array}
$$

Unhumified Sphagnum peat dating end of clearance episode.

\section{White Moss series}

Peat samples are from two exposed peat faces at White Moss, $15 \mathrm{~km} \mathrm{~N}$ of Barrow-in-Furness, Cumbria $\left(54^{\circ} 15^{\prime} \mathrm{N}, 3^{\circ} 13^{\prime} \mathrm{W}\right)$. Samples were coll from peat faces and as sub-samples from series of monoliths from sites as part of strat and palynol study. Coll 1981 by G T Wimble and E W Cloutman, and subm 1983 by GTW. Comment (GTW): at 1st site, WMN (Natl Grid Ref SD 216855), Monolith WMN1 was taken at surface. Monolith WMN3 was taken $1.5 \mathrm{~m}$ to $\mathrm{N}$ of $\mathrm{WMN} 1$ and $20 \mathrm{~cm}$ lower $(40 \mathrm{~cm}$ from surface). Mo- 
nolith WMN2 was taken $2 \mathrm{~m}$ to $\mathrm{N}$ of WMN3 and at same depth as WMN1. Monolith WMS1 was taken at 2nd site $1 \mathrm{~km}$ SW of $1 \mathrm{st}$ (Natl Grid Ref SD 221848 ) at depth $10 \mathrm{~cm}$ below present bog surface.

CAR-701. WMN1 15-17cm $730 \pm 60$

Moderately unhumified Sphagnum peat taken in lowest few $\mathrm{cm}$ of recurrence peat.

CAR-704. WMN1 41-42cm

$$
\begin{array}{r}
1770 \pm 60 \\
\delta^{13} C=-26.9 \%
\end{array}
$$

Moderately unhumified Sphagnum peat dating recurrence surface and end of clearance episode.

CAR-700. WMN1 49-50cm

$$
\begin{array}{r}
\mathbf{1 8 6 0} \pm \mathbf{6 0} \\
\delta^{13} C=-27.0 \% \%
\end{array}
$$

Moderately unhumified Sphagnum peat dating change from more to less humified peat, and max clearance of forest as indicated by high NAP values.

CAR-726. WMN1 55-56cm

$$
\begin{array}{r}
\mathbf{2 1 3 0} \pm \mathbf{7 0} \\
\delta^{13} \mathrm{C}=-26.4^{0} \%
\end{array}
$$

Muddy Sphagnum peat dating top of rather humified Sphagnum/Calluna hummock.

\section{CAR-727. WMN1 67-68cm}

$$
2320 \pm 70
$$

Unhumified Sphagnum peat dating approx start of clearance episode.

\section{CAR-728. WMN1 97-98cm}

$$
\begin{array}{r}
\mathbf{3 0 0 0} \pm \mathbf{7 0} \\
\delta^{13} \mathrm{C}=-27.5 \% 0
\end{array}
$$

Slightly humified peat dating base of possible retardation layer and end of clearance episode.

\section{CAR-699. WMN2 16-17cm}

$$
\delta^{13} C=\begin{array}{r}
\mathbf{8 4 0} \pm \mathbf{6 0} \\
-26.7 \%
\end{array}
$$

Moderately humified Sphagnum peat dating top of retardation layer and beginning of clearance episode.

\section{CAR-703. WMN2 36-38cm}

$1630 \pm 60$

Moderately humified Sphagnum peat dating top of retardation layer and max forest regeneration between two clearance episodes.

CAR-695. WMN2 51-52 cm

$$
\begin{array}{r}
\mathbf{2 0 7 0} \pm \mathbf{6 0} \\
\delta^{I 3} C=-26.8 \% 0
\end{array}
$$

Humified Sphagnum peat dating top of retardation layer and max clearance of forest as indicated by high NAP values. 
CAR-696. WMN2 73-74cm

$$
2690 \pm 70
$$

Moderately unhumified Sphagnum peat dating possible recurrence peat.

CAR-697. WMN2 93-94cm

$$
\begin{array}{r}
\mathbf{2 9 4 0} \pm \mathbf{7 0} \\
\delta^{13} C=-25.8 \% \text { o }
\end{array}
$$
episode.

Moderately unhumified Sphagnum peat taken near end of clearance

CAR-698. WMN2 103-104cm

$$
\begin{array}{r}
\mathbf{3 2 4 0} \pm \mathbf{7 0} \\
\delta^{13} C=-26.7 \% \text { o }
\end{array}
$$

Moderately humified Sphagnum peat dating start of clearance episode. de.

CAR-731. WMN3 24-25cm

$$
1850 \pm 70
$$

Unhumified Sphagnum peat dating base of retardation layer.

CAR-729. Sample B

$$
\begin{array}{r}
1470 \pm 60 \\
\delta^{13} C=-26.7 \% 0
\end{array}
$$

Fairly humified Sphagnum peat from $40 \mathrm{~cm}$ N of Monolith WMN3 at depth $50 \mathrm{~cm}$ dating top of retardation layer.

\section{CAR-730. Sample D}

$2210 \pm 70$

$\delta^{13} \mathrm{C}=-25.7 \%$

Muddy Sphagnum peat from $40 \mathrm{~cm}$ N of Monolith WMN3 at depth $80 \mathrm{~cm}$ dating possible base of retardation layer.

\section{CAR-680. WMS1 18-19cm}

$390 \pm 60$

$\delta^{13} C=-25.9 \%$ surface.

Moderately unhumified Sphagnum peat dating possible recurrence

CAR-693. WMS1 28-29cm

$$
940 \pm 60
$$

Unhumified Sphagnum peat dating start of clearance episode.

CAR-694. WMS1 39-40cm

$1160 \pm 60$

Moderately humified Sphagnum peat dating max forest clearance as indicated by high NAP values.

CAR-702. WMS1 50-51 cm

$1570+60$

$\delta^{13} C=-27.5 \%$

Moderately humified Sphagnum peat dating probable recurrence surface and start of clearance episode. 
CAR-681. WMS1 67-68cm

$2010 \pm 60$

$\delta^{13} C=-26.8 \%$

Muddy peat with Menyanthes rhizomes dating recurrence peat and max clearance of forest as indicated by high NAP values.

CAR-682. WMS1 80-81 cm

$$
\begin{array}{r}
\mathbf{2 4 9 0} \pm \mathbf{7 0} \\
\delta^{13} \mathrm{C}=-26.7 \% 0
\end{array}
$$
sode.

Muddy peat with charcoal fragments dating start of clearance epi-

\section{Greece}

\section{Sigitikos series}

Samples are sediments from sapropelic horizons within hemipelagic sediments in Sigitikos Gulf, N Greece. Samples are sub-samples of cores taken in sea depths from 315 to $992 \mathrm{~m}$. Coll 1978 by C Perissoratis, Inst Geol and Mineral Exploration, Athens, during marine geol research cruise. Subm 1980 by M Brooks, Dept Geol, Univ Coll, Cardiff. Comment: acid wash pretreatment.

CAR-368. AG1 58-111

$$
\begin{array}{r}
\mathbf{8 2 1 0} \pm \mathbf{9 0} \\
\delta^{13} C=-24.4 \% 0
\end{array}
$$

Sapropelic layer at 58 to $111 \mathrm{~cm}$ from Core AG1, length $175 \mathrm{~cm}$, taken $7 \mathrm{~km} \mathrm{~W}$ of Ammuliani Is. $\left(40^{\circ} 15^{\prime} 00^{\prime \prime} \mathrm{N}, 23^{\circ} 50^{\prime} 27^{\prime \prime} \mathrm{E}\right)$ at water depth $315 \mathrm{~m}$.

CAR-369. AG3 113-153

$$
\begin{array}{r}
8590 \pm 100 \\
\delta^{13} C=-24.4 \%
\end{array}
$$

Sapropelic layer at 113 to $153 \mathrm{~cm}$ from Core AG3, length $184 \mathrm{~cm}$, taken $4.5 \mathrm{~km} \mathrm{~W}$ of peninsula of Sithonia $\left(40^{\circ} 11^{\prime} 50^{\prime \prime} \mathrm{N}, 23^{\circ} 53^{\prime} 10^{\prime \prime} \mathrm{E}\right)$ near basin of ca $400 \mathrm{~m}$ depth. Core taken at water depth $372 \mathrm{~m}$.

CAR-370. AG8 95-139

$$
\begin{array}{r}
\mathbf{8 6 0 0} \pm \mathbf{1 1 0} \\
\delta^{13} C=-24.2^{\%} \%
\end{array}
$$

Sapropelic layer at 95 to $139 \mathrm{~cm}$ from Core AG8, length $180 \mathrm{~cm}$, taken in middle of mouth of Gulf of Sigitikos $\left(40^{\circ} 05^{\prime} 15^{\prime \prime} \mathrm{N}, 24^{\circ} 09^{\prime} 11^{\prime \prime} \mathrm{E}\right)$ at water depth $396 \mathrm{~m}$.

\section{CAR-371. NA25 120-165}

$10,140 \pm 110$

$\delta^{13} C=-24.6 \%$

Sapropelic layer at 120 to $165 \mathrm{~cm}$ from Core NA25, length $176 \mathrm{~cm}$, taken $10 \mathrm{~km} \mathrm{SE}$ of Athos peninsula $\left(40^{\circ} 05^{\prime} 55^{\prime \prime} \mathrm{N}, 24^{\circ} 29^{\prime} 30^{\prime \prime} \mathrm{E}\right)$ at water depth $992 \mathrm{~m}$. 
Wales

\section{Magor series}

Samples are from coffin buried $2 \mathrm{~m}$ under present surface, within and sealed by estuarine silt and deposited under conditions of complete inundation, excavated 1978 by G Dowdell (1978), GGAT, at Magor, 13km ESE of Newport, Gwent $\left(51^{\circ} 34^{\prime}\right.$ N, $2^{\circ} 49^{\prime}$ W, Natl Grid Ref ST 436850). Coll 1978 by GD and subm by GGAT. Site $1 \mathrm{~km}$ from present HWM in Caldicot levels and close to site of Roman finds (Boon, 1976).

\section{CAR-198. Magor 1}

$$
\begin{array}{r}
1660 \pm 60 \\
\delta^{13} C=-20.1 \% \text { o }
\end{array}
$$

Collagen from human bone from within coffin id by T P O'Connor, Dept Archaeol, Univ Coll, Cardiff.

CAR-199. Magor 2

$$
1570 \pm 70
$$

Wood from coffin.

\section{Llandough series}

Samples are human bone from burials on site of Roman villa excavated 1979 by H S Owen-John for GGAT at Llandough, $5 \mathrm{~km} \mathrm{SW}$ of Cardiff, S Glamorgan ( $51^{\circ} 27^{\prime} \mathrm{N}, 3^{\circ} 12^{\prime} \mathrm{W}$, Natl Grid Ref ST 168731). Site comprised Roman villa with some Iron age antecedents and with Medieval and later occupations. Samples taken to establish period of burials. Coll 1979 by A D Russel and subm by GGAT.

CAR-271. Sample 25/052

$$
\delta^{13} C=\begin{array}{r}
\mathbf{8 9 0} \pm \mathbf{6 0} \\
-20.9 \%
\end{array}
$$

Collagen from human bone from grave dug through $\mathrm{W}$ wall of Room $\mathrm{M}$ of Roman bldg and demolition rubble.

\section{CAR-305. Sample 25/122}

$1160 \pm 70$

$\delta^{13} \mathrm{C}=-20.9 \%$

Collagen from human bone from one of series of graves dug into natural subsoil within area of Medieval village to NW of Roman bldg.

CAR-306. Sample 25/145

$$
\begin{array}{r}
1180 \pm 60 \\
\delta^{13} C=-20.9 \% 0
\end{array}
$$

Collagen from human bone from one of series of graves dug into natural subsoil within area of Medieval village to NW of Roman bldg.

\section{Biglis series}

Bone samples are from late Iron age to Romano British farmstead excavated 1978 to 1979 by J Parkhouse, GGAT, at Biglis, $1 \mathrm{~km}$ E of Barry, S Glamorgan (51 $25^{\prime} \mathrm{N}, 3^{\circ} 14^{\prime} \mathrm{W}$, Natl Grid Ref ST 142694). Coll 1978 and 
1979 by JP and subm by GGAT. Three construction phases were id. Unenclosed settlement was followed by double enclosure with double palisade. Third phase comprised replacement of palisade by bank. Human burials from 3rd phase were cut through bank.

CAR-269. Sample 21/270

$$
\begin{array}{r}
1830 \pm 70 \\
\delta^{13} C=-20.6 \% 0
\end{array}
$$

Collagen from human bone from grave, partially lined with stone slabs, cut through inner palisade trench surrounding site. Dates time of palisade disuse.

CAR-270. 21/387

$1520 \pm 70$
$\delta^{13} C=-21.0 \%$

Collagen from human bone from slab-lined grave constructed within corn drying kiln. Grave postdates construction of stone boundary bank and possibly postdates bank disuse.

CAR-307. Sample 21/537

$$
\begin{array}{r}
1980 \pm 70 \\
\delta^{13} C=-22.2 \% 0
\end{array}
$$

Collagen from animal bone from rubbish pit used in 2nd phase of settlement and underlying bank of 3rd phase.

CAR-308. Sample 21/544

$$
1870 \pm 70
$$

Collagen from animal bone from rubbish pit used in 2nd phase of settlement and underlying bank of $3 \mathrm{rd}$ phase.

\section{Coed-y-Cymdda series}

Samples are from hill slope enclosure excavated 1978 and 1979 by H S Owen-John for GGAT, at Coed-y-Cymdda, $6.5 \mathrm{~km} \mathrm{SW}$ of Cardiff, S Glamorgan $\left(51^{\circ} 27^{\prime} \mathrm{N}, 3^{\circ} 15^{\prime} \mathrm{W}\right.$, Natl Grid Ref ST 133740). Coll 1978 and 1979 by D Russel, GGAT, and subm by GGAT.

\section{CAR-205. Object 053/1047}

$\mathbf{2 6 0 0} \pm \mathbf{7 0}$

Collagen from antler from lower fill of $\mathrm{S}$ ditch terminal of $\mathrm{E}$ entran to enclosure, dating silting of enclosure ditch.

\section{CAR-206. Object 053/1046}

$2250 \pm 70$

Collagen from human bones from lower fill of $S$ ditch terminal at $\mathrm{E}$ entrance of enclosure, dating silting of enclosure ditch.

\section{CAR-207. Object 054/1044}

$2680 \pm 70$

$$
\delta^{13} C=-21.7 \%
$$

Collagen from antler and bone from lower fill of $\mathrm{N}$ ditch terminal of $\mathrm{E}$ entrance of enclosure, dating silting of ditch. 


\section{CAR-304. Object 079/1118}

$$
\begin{array}{r}
3110 \pm 70 \\
\delta^{13} C=-26.3 \% 0
\end{array}
$$

Charcoal from occupation horizon stratified beneath main enclosure bank of site, antedating bank construction.

General Comment (HSOJ): site was first cleared in late Neolithic period and was occupied in late Bronze age as attested by pottery fragments and CAR304. CAR-205 and -207 are earlier than expected from pottery remains from same context; date for CAR-206 is more consistent.

\section{Ardudwy series}

Charcoal samples are from two circular enclosure sites excavated 1980 to 1981 by R Kelly, GAT, in Ardudwy. Moel-y-Gerddi lies $3.5 \mathrm{~km}$ ENE of Harlech, Gwynedd (52 $52^{\prime} \mathrm{N}, 4^{\circ} 03^{\prime} \mathrm{W}$, Natl Grid Ref SH 617317) and embraced 2 phases of construction probably belonging to single period of occupancy. First phase was characterized by slot ca $30 \mathrm{~m}$ across supporting wooden palisade which surrounded central house. Second phase followed same construction lines but in stone. Remnant magnetic date for last firing of hearth stone in second phase estimated date at 100-200 BC. Other site ca $1 \mathrm{~km} \mathrm{NW}$ at Erw-wen was very similar. Coll 1980 and 1981 by RK and subm by GAT. Peat core samples were also taken (see Geologic Samples, above).

CAR-397. Moel-y-Gerddi 80 SO28

$$
\begin{array}{r}
\mathbf{4 5 9 0} \pm \mathbf{8 0} \\
\delta^{13} C=-27.5 \% 0
\end{array}
$$

Charcoal from fill of hearth pit sealed by and antedating 2nd phase stone wall.

\section{CAR-398. Moel-y-Gerddi 80 SO34}

$$
\begin{array}{r}
2350 \pm 70 \\
\delta^{13} C=-26.1 \% 0
\end{array}
$$

Charcoal from fill of pit surrounding central hearth in 2nd phase house dating end of house use. Hearth stone dated magnetically.

CAR-525. Moel-y-Gerddi 80 SO13/15/23

$4760 \pm 70$

Charcoal from primary fill of 1 st phase palisade slot, possibly representing remains of timber used in palisade construction.

$2250 \pm 110$

CAR-526. Moel-y-Gerddi 80 SO16(i) + SO16/21 $\delta^{13} C=-23.7 \%$

Charcoal from fill of post hole sealed by stone wall dating 1 st phase and antedating 2 nd.

CAR-527. Moel-y-Gerddi 80 SO28(ii)

$4540+70$

$\delta^{13} C=-25.9 \%$

Charcoal from fill of hearth pit sealed by 2 nd phase stone wall. Duplicates CAR-397. 
CAR-528. Moel-y-Gerddi 80 SO33

$\mathbf{4 0 3 0} \pm \mathbf{8 0}$

Charcoal from fill of hearth sealed by 2nd phase stone house floor. Hearth stone dated magnetically to beyond present range for this method.

\section{CAR-529. Moel-y-Gerddi 80 SO34(ii)}

$2290 \pm 70$

Charcoal from pit adjacent to central hearth stone in 2nd phase stone house. Duplicates CAR-398.

CAR-530. Erw-wen 81 SO01

$2410 \pm 60$

Charcoal from occupation surface colluvially formed against inside face of main enclosure wall, and sealed by further colluvium. Should provide latest date for occupation of site.

\section{CAR-531. Erw-wen 81 SO04}

$$
2660 \pm 60
$$$$
\delta^{13} C=-26.1 \%
$$

Charcoal from shallow deposit comprising remains of hearth pit dug into floor of stone phase house and sealed by later surface laid after abandonment of house.

\section{CAR-532. Erw-wen 81 SO09}

$$
\begin{array}{r}
2470 \pm 70 \\
\delta^{13} C=-26.0 \% 0
\end{array}
$$

Charcoal from fill of 1 st phase timber house wall slot. Slot had been deliberately filled in and whole site levelled prior to bldg of 2nd phase stone house.

\section{Cyfannedd series}

Charcoal samples from hut circle site excavated 1978 by $\mathrm{P}$ Crew, Snowdonia Natl Park Study Centre, Gwynedd, at Cyfannedd, $11 \mathrm{~km}$ WSW of Dolgellau, Gwynedd (52 $41^{\prime} \mathrm{N}, 4^{\circ} 01^{\prime} \mathrm{W}$, Natl Grid Ref SH 633117). Site, on $\mathrm{N}$ facing shelf at alt $370 \mathrm{~m}$ on slope of Cader Idris, consists of single hut circle with 3 phases of activity. Coll 1978 by PC and subm by GAT.

CAR-418. Cyfannedd Sample 1

$2010 \pm 70$ $\delta^{13} C=-27.4 \%$

Charcoal from concentration within matrix of burned clay and stone sealed by Phase 3 cairn material.

CAR-419. Cyfannedd Sample 2 $1970+70$

Charcoal from upper part of hearth of Phase 1 hut circle, partly sealed by clay floor of Phase 2 hut circle.

CAR-420. Cyfannedd Sample 3

$$
\begin{array}{r}
1740 \pm 70 \\
\delta^{13} C=-27.2 \% 0
\end{array}
$$

Charcoal from hearth of Phase 1 hut circle, partly sealed by clay floor of Phase 2 hut circle. 


\section{Gwernvale series}

Charcoal samples from Neolithic chambered tomb on kame terrace on N side of Usk Valley, excavated 1977 to 1978 by W J Britnell $(1979 ; 1980)$, CPAT, at Gwernvale, $0.5 \mathrm{~km} \mathrm{~W}$ of Crickhowell, Powys $\left(51^{\circ} 52^{\prime} \mathrm{N}, 3^{\circ} 09^{\prime} \mathrm{W}\right.$, Natl Grid Ref SO 211192). Coll 1977, 1978 by WJB and subm by CPAT.

\section{CAR-113. Pit F68}

$\mathbf{5 0 5 0} \pm \mathbf{8 0}$

Charcoal from upper layer of pit beneath cairn in dark ashy soil, assoc with early Neolithic pottery. Dates pre-cairn activity.

CAR-114. Pit F58

$4390 \pm 70$

Charcoal from various depths in pit outside Chamber 2, assoc with late Neolithic pottery. Antedates closure of cairn.

\section{CAR-116. Pit F47}

$$
4590 \pm 80
$$

Charcoal from various depths in pit outside Chamber 2, assoc with late Neolithic pottery. Antedates closure of cairn.

\section{CAR-118. Pit F308}

$$
6900 \pm 80
$$

Charcoal from pit beneath outer revetment wall of long cairn. Comment (WJB): no assoc artifacts but possibly dates late Mesolithic activity.

\section{Dinorben series}

Charcoal and bone samples are from hillfort excavated 1977 to 1978 by G Guilbert, CPAT, at Dinorben (Gardner \& Savory, 1964; Savory, 1971; Guilbert, $1979 ; 1980 \mathrm{a}, \mathrm{b}) 2.5 \mathrm{~km}$ SE of Abergele, Clwyd $\left(53^{\circ} 15^{\prime} \mathrm{N}\right.$, $3^{\circ} 33^{\prime} \mathrm{W}$, Natl Grid Ref SH 968757). Coll 1977, 1978 by GG and subm by CPAT. Floral id by G Hillman, Dept Plant Sci, Univ Coll, Cardiff, and faunal id by B Noddle, Dept Anatomy, Univ Coll, Cardiff.

\section{CAR-119. B300(J)}

$2390 \pm 45$

Corylus charcoal from discrete branch burned in situ in early timberlaced rampart.

\section{CAR-120. B300(K)}

$$
\begin{array}{r}
\mathbf{2 4 1 0} \pm \mathbf{6 0} \\
\delta^{13} C=-25.6 \%
\end{array}
$$

Fraxinus excelsior charcoal from discrete branch burned in situ in early timber-laced rampart.

\section{CAR-121. B300(Q)}

$2410 \pm 60$

Fraxinus excelsior charcoal from discrete branch burned in situ in early timber-laced rampart. 
CAR-122. B300(U)

$\mathbf{2 4 5 0} \pm \mathbf{6 0}$ laced rampart.

CAR-123. B287(3)

$$
\begin{array}{r}
\mathbf{2 4 3 0} \pm \mathbf{6 0} \\
\delta^{13} C=-25.3 \% 0
\end{array}
$$

Charred inner 20 rings of Quercus fragment with no bark apparent, from burned floor deposit on quarried Platform 38. Postdates earliest bldg of Bank 1 and antedates 2nd.

CAR-124. B287(5)

$$
\begin{array}{r}
\mathbf{2 3 6 0} \pm \mathbf{6 0} \\
\delta^{13} C=-25.7 \% 0
\end{array}
$$

Charred twig fragments from scatter through burned floor deposit on Platform 38, postdating earliest bldg of Bank 1 and antedating 2nd.

\section{CAR-125. B184}

$$
\begin{array}{r}
\mathbf{2 3 0 0} \pm \mathbf{6 0} \\
\delta^{13} C=-26.0 \% 0
\end{array}
$$

Charcoal from thin spread of sooty soil surrounding clay hearth at center of stake-built structure on Platform 39 and contemporary with usage of structure. Sample coll by flotation of deposit.

CAR-126. B29

$$
\begin{array}{r}
\mathbf{2 3 1 0} \pm \mathbf{6 0} \\
\delta^{13} C=-26.3 \%
\end{array}
$$

Charcoal from fill of shallow pit overlain by topsoil. Pit cut through by post hole at entrance to curvilinear ditched structure inside hillfort.

CAR-128. B343

$$
\begin{array}{r}
\mathbf{2 5 0 0} \pm \mathbf{7 0} \\
\delta^{13} C=-21.8 \%
\end{array}
$$
Ditch 1, antedating early rampart.

CAR-129. B352b

$1920 \pm 60$

Collagen from bones of cattle from basal $0.5 \mathrm{~m}$ of rubble fill of Ditch 3 , dating dereliction of outer defenses.

CAR-130. B352b

$$
\begin{array}{r}
1480 \pm 60 \\
\delta^{13} C=-22.0 \% 0
\end{array}
$$

Collagen from bones of cattle from rubble fill of Ditch 3 at 0.5 to $1 \mathrm{~m}$ above ditch floor and above level of CAR-129.

\section{CAR-131. B348b}

$$
\begin{array}{r}
2170 \pm 60 \\
\delta^{13} C=-22.0 \%
\end{array}
$$
Bank 2.

Collagen from bones of cattle from fine-grained silt deposit under 
CAR-132. B348b

Collagen from bones of cattle from fine-grained silt deposit under Bank 2, duplicating CAR-131.

CAR-133. B346

$\mathbf{1 8 1 0} \pm \mathbf{5 0}$

$\delta^{13} C=-22.4 \%$

Collagen from bones of sheep and cattle from fill of Ditch 2.

CAR-167. B317 \& B318

$\mathbf{2 4 7 0} \pm \mathbf{6 0}$

$\delta^{13} C=-24.6 \%$

Collagen from bones of cattle and roe deer from occupation horizon at surface of silty soil under Bank 1, antedating earliest bldg of Bank 1 .

CAR-203. B352b

$1600 \pm 70$

Collagen from bones of cattle, sheep, pig, and dog from same level of Ditch 3 as CAR-130. Comment: $\delta^{13} \mathrm{C}$ value of $-22 \% \pm 2$ used in date calculation.

CAR-204. B352a

$$
\begin{array}{r}
\mathbf{1 5 5 0} \pm \mathbf{5 0} \\
\delta^{13} C=-21.8 \% \text { o }
\end{array}
$$

Collagen from bones of cattle from rubble fill of Ditch 3 at 1 to $1.5 \mathrm{~m}$ above ditch floor and above level of CAR-130 and -203.

\section{Rhuddlan series}

Charcoal and bone samples from town ditch and bank excavated 1979 by J F Manley, Clwyd Co Council, ca $1 \mathrm{~km} \mathrm{~S}$ of Rhuddlan, Clwyd $\left(53^{\circ} 17^{\prime} \mathrm{N}\right.$, $3^{\circ} 27^{\prime}$ W, Natl Grid Ref SJ 030773). Coll 1979 by JFM; subm by CPAT.

CAR-239. Sample 1

$$
510 \pm 60
$$

Collagen from bones and teeth of Bos and Ovis id by B Noddle, from primary silt fill of main ditch.

CAR-240. Sample 2

$$
\begin{array}{r}
1160 \pm 60 \\
\delta^{13} C=-26.2^{\%} \%
\end{array}
$$

Charcoal. Fragments of Quercus and some Ulex id by G Hillman, from middle of outer bank underlying topmost layer of bank. Sample coll as discrete lumps on site.

\section{CAR-241. Sample 3}

$$
\begin{array}{r}
1160 \pm 60 \\
\delta^{13} C=-26.4 \% o
\end{array}
$$

Charcoal fragments of Quercus and Ulex id by G Hillman from middle of outer bank beneath topmost layer. Sample obtained by flotation of bulk deposit on site. 
Trelystan series

Charcoal samples are from two adjacent round barrows excavated 1979 by W J Britnell $(1981 ; 1982)$, at Trelystan, Long Mt, 8km E of Welshpool, Powys (52 $39^{\prime} \mathrm{N}, 3^{\circ} 04^{\prime} \mathrm{W}$, Natl Grid Ref SJ 277070). Coll 1979 by WJB and subm by CPAT. Site comprised late Neolithic settlement superseded by Bronze age burials and round barrows.

\section{CAR-272. Object 1006 Context D 8}

$4260 \pm 70$

Charcoal from Pit 13 within pre-barrow stake-built Structure B dating pre-barrow settlement, antedating Barrow I.

\section{CAR-273. Object 1007 Context D 7}

$$
\begin{array}{r}
4140 \pm 70 \\
\delta^{13} C=-25.7 \% 00
\end{array}
$$

Charcoal from Pit 14 within stake-built Structure B dating pre-barrow settlement, antedating Barrow I.

\section{CAR-274. Object 946 Context D 6}

$$
\begin{array}{r}
3990 \pm 70 \\
\delta^{13} C=-25.1 \% 0
\end{array}
$$

Charred hazel nutshells from surface of hearth within stake-built Structure B dating pre-barrow settlement, antedating Barrow I.

CAR-275. Object 325 Context C 16

$$
\begin{array}{r}
4050 \pm 70 \\
\delta^{13} C=-26.2 \%
\end{array}
$$

Charcoal from Pit 1 within stake-built Structure A dating pre-barrow settlement.

\section{CAR-276. Object 350 Context C 21}

$3960 \pm 70$

$\delta^{13} C=-26.2 \%$

Corylus Avellana charcoal id by G Hillman, from slot adjacent to hearth within stake-built Structure A.

\section{CAR-277. Object 538 Context BI 22}

$$
\begin{array}{r}
3450 \pm 70 \\
\delta^{13} C=-26.1 \% 0
\end{array}
$$

Charred wood and hazel nuts from Pit 18 antedating enlargement of Barrow I. Comment: date previously reported as $3455 \pm 70$ (Britnell, 1981).

CAR-278. Object 337 Context BI 10

$$
\begin{array}{r}
\mathbf{3 5 0 0} \pm \mathbf{6 0} \\
\delta^{13} C=-26.2 \% 0
\end{array}
$$

Charcoal from area of burning beneath enlargement of Barrow I and postdating early settlement phase.

\section{CAR-279. Object 620 Context BI 25}

$$
\begin{array}{r}
3750 \pm 70 \\
\delta^{13} C=-26.0 \%
\end{array}
$$

Charcoal from large burned area on ground surface beneath enlargement of Barrow I. 
CAR-280. Object 507, Context BI 21

Charcoal from Stake 5 of setting accompanying Barrow I, Burial 4.

\section{CAR-281. Object 335 Context BI 16}

$3700+70$

Charcoal from burned $\log$ assoc with cremation burial Barrow I, Burial 4, dating enlargement of Barrow I.

\section{CAR-282. Object 446 Context BII 4}

$4350 \pm 70$

Charcoal from within rubble fill of pit grave of Barrow II, Burial 1.

\section{CAR-283. Object 148 Context BII 2}

$3550 \pm 60$

Charcoal from cremation material in enlarged food vessel in Barrow II, Burial 3. Dates later use of Barrow II.

\section{CAR-285. Object 603 Context BI 19}

$3540 \pm 70$

Charcoal from significant spread on stabilized surface of early phase of Barrow I.

\section{CAR-390. Object 843 Context BII 13}

$3550 \pm 70$

Charcoal from thin layer on surface of buried soil beneath Phase 2 of Barrow II providing approx construction date.

\section{CAR-290. Church Street}

$1770 \pm 70$

$$
\delta^{13} \mathrm{C}=-27.4 \%
$$

Outer 12-yr growth of Quercus branch with bark from top of 1st phase defensive ditch and sealed by 2nd phase of Roman town defenses, excavated 1978 by $\mathrm{H}$ J James, DAT, at Church St, Carmarthen, Dyfed ( $51^{\circ} 51^{\prime}$ N, $4^{\circ} 18^{\prime}$ W, Natl (rrid Ref SN 415201). Coll 1978 by HJJ and subm by DAT.

\section{CAR-291. Caer Bayvil}

$1290 \pm 60$

Collagen from human bone from cist grave at Iron age enclosure and Early Christian cemetery excavated 1979 by H J James, at Caer Bayvil, 8km SW of Cardigan, Dyfed (52 $02^{\prime} \mathrm{N}, 4^{\circ} 45^{\prime} \mathrm{W}$, Natl Grid Ref SN 112417$)$. Coll 1979 by $\mathrm{HJJ}$ and subm by DAT. Comment $(\mathrm{HJJ})$ : sample dated to determine if grave was Christian.

\section{CAR-315. Longstone Field}

$$
\begin{array}{r}
3310 \pm 70 \\
\delta^{13} C=-26.4^{\prime \prime} \% 0
\end{array}
$$

Charcoal, possibly from heartwood of Quercus branch from pit on site comprising standing stone, stake-built structures and pits, excavated 1979 
by D Benson and G Williams, DAT, at Longstone Field, St Ishmael's $6 \mathrm{~km}$ W of Milford Haven, Dyfed (51 $44^{\prime} \mathrm{N}, 5^{\circ} 07^{\prime} \mathrm{W}$, Natl Grid Ref SM 848084). Coll 1979 by DB and GW and subm by DAT.

\section{St John's Priory series}

Charcoal samples recovered by flotation are from Early Christian and Medieval monastery excavated 1979 by T James, at St John's Priory, Carmarthen, Dyfed (51 $51^{\prime}$ N, $4^{\circ} 18^{\prime} \mathrm{W}$, Natl Grid Ref SN 419204). Coll 1979 by TAJ and subm by DAT.

\section{CAR-288. Ditch}

$1220 \pm 60$

Charcol from primary silt fill of Ditch 234 dating series of ditches beneath later Medieval cemetery.

\section{CAR-289. Lime kiln}

$$
\delta^{13} C=\begin{array}{r}
\mathbf{8 7 0} \pm \mathbf{6 0} \\
-26.5 \% 0
\end{array}
$$

Charcoal from basal fill of lime kiln antedating extension to Priory church and some Medieval graves.

\section{Corn Du series}

Peat samples are from mountain-top cist constructed directly on undisturbed peat surface (Crew, 1978) excavated 1978 by P Crew, at Corn Du Mt, 8km SW of Brecon, Powys $\left(51^{\circ} 52^{\prime} \mathrm{N}, 3^{\circ} 26^{\prime} \mathrm{W}\right.$, Natl Grid Ref SO 007213 ) alt $873 \mathrm{~m}$. Coll 1978 by D Roe; subm by CPAT. Comment: samples pretreated to remove fulvic and humic materials.

\section{CAR-201. Sample SO4}

$$
\begin{array}{r}
\mathbf{3 8 0 0} \pm \mathbf{8 0} \\
\delta^{13} C=-26.9 \% \text { o }
\end{array}
$$

Calluna fragments id by B Smith, from uppermost $1.5 \mathrm{~cm}$ layer of yellow-green peat at pre-cairn surface sealed by redeposited peat of cairn structure.

\section{CAR-202. Sample SO5}

$$
\mathbf{3 7 0 0} \pm \mathbf{8 0}
$$

Calluna fragments id by BS from uppermost $1 \mathrm{~cm}$ layer of undisturbed peat sealed by large basal slab of cist.

\section{Moel Goedog Circle I series}

Charcoal samples are from pits at early Bronze age ring cairn excavated 1978 by F M Lynch, Univ Coll, Bangor, at Moel Goedog, 3km NE of Harlech, Gwynedd (52 $52^{\prime} \mathrm{N}, 4^{\circ} 04^{\prime} \mathrm{W}$, Natl Grid Ref SH 610324). Coll 1978 by FML; subm by GAT. Comment (FML): site, 1 of 2 on either side of Bronze age trackway, lies at alt $270 \mathrm{~m}$ on open $\mathrm{mt}$ side. Twelve upright stones, of which 3 remain, were placed in circle backed by low ring of cairn material on level terrace cut into hillside. Within central area were 10 pits, most of which contained dark earth and charcoal. Some pits contained urns and cremated bones (Lynch, in press a). 
CAR-160. SS9a \& SS9

Charcoal from fill of Pit F8, containing food vessel urn.

CAR-161. SS7

$3450 \pm 70$

Charcoal from fill of Pit F5 at center of circle.

$$
\delta^{13} C=-25.7 \%
$$

CAR-162. SS15

$3610 \pm 70$

$\delta^{13} \mathrm{C}=-26.7 \%$

Charcoal from fill of Pit F11 close to edge of central area and covered by flat stone and collapsed cairn material.

CAR-163. SS6

$$
\begin{array}{r}
\mathbf{3 6 0 0} \pm \mathbf{7 0} \\
\delta^{13} C=-25.7 \% \text { o }
\end{array}
$$

Charcoal from fill of Pit F1 in central area and covered by stone slabs. Pit also contained small quantity of bone.

CAR-164. SS5

$3470 \pm 70$

$\delta^{13} C=-25.2 \%$ stones.

Charcoal from small pit in central area, Pit F3, covered by spread of

CAR-165. SS13

$$
\begin{array}{r}
\mathbf{3 6 4 0} \pm \mathbf{7 0} \\
\delta^{13} C=-25.6 \% 0
\end{array}
$$

Charcoal from within and around mouth of cremation urn from base of Pit F10.

CAR-166. SS16

$$
\begin{array}{r}
\mathbf{3 4 7 0} \pm \mathbf{7 0} \\
\delta^{13} C=-25.0 \% 0
\end{array}
$$

Charcoal from immediately around remains of broken urn in Pit F7.

General Comment (FML): dates fall in expected range and confirm early Bronze date for this type of site. Three strat phases noted during excavation implied possible spread over longer period than results indicated. Although dates span nearly $200 \mathrm{yr}$, their sequence does not appear to conform to that of strat though this does not deny field observations.

\section{Cefn Caer Euni series}

Charcoal samples are from early Bronze age kerb circle excavated 1971 by F M Lynch, at Cefn Caer Euni, $7 \mathrm{~km} \mathrm{SW}$ of Corwen, Merioneth $\left(52^{\circ} 57^{\prime} \mathrm{N}, 3^{\circ} 30^{\prime} \mathrm{W}\right.$, Natl Grid Ref SH 994410$)$. Coll 1971 and subm 1982 by FML. Site was on rocky peat-covered ridge and comprised ritual monument with underlying occupation horizon containing "Domestic Beaker" sherds and charcoal (Lynch, in press b).

\section{CAR-600. Sample A}

$$
\begin{array}{r}
4130 \pm 70 \\
\delta^{13} C=-25.9 \% 0
\end{array}
$$

Charcoal from settlement layer, trodden into gray-blue horizon assoc with "Domestic Beaker" sherds; horizon was covered by sterile yellow clay before construction of monument. Provides date for settlement. 
CAR-601. Sample B

$3380 \pm 70$

Charcoal from pit within false portal of monument and related to use of kerb circle.

General Comment (FML): date for monument is within expected range but date for settlement is earlier than expected, both because it was thought that occupation had been rapidly succeeded by erection of monument and because pottery had been id as Beaker. However, it is not classic and should perhaps be reconsidered.

\section{Carreg Coetan series}

Charcoal samples are from Neolithic chambered tomb in Afon Nyfer valley $12 \mathrm{~km}$ E of Fishguard, Dyfed $\left(52^{\circ} 01^{\prime} \mathrm{N}, 4^{\circ} 50^{\prime} \mathrm{W}\right.$, Natl Grid Ref SN 060394). Site, excavated 1979 and 1980 by S E Rees, Ancient Monuments Branch, Welsh Office, comprised mound of redeposited subsoil surrounded by stone kerb. Coll 1979, 1980 and subm 1980 by SER.

\section{CAR-391. Sample 1}

$4560 \pm 80$

Charcoal from old ground surface sealed beneath mound material, antedating erection of mound.

\section{CAR-392. Sample 2}

$4830 \pm 80$

Charcoal from area of burning, Area F36, sealed beneath stone kerb, antedating erection of kerb.

CAR-393. Sample 3

$$
\begin{array}{r}
\mathbf{4 4 7 0} \pm \mathbf{8 0} \\
\delta^{13} C=-26.8 \%
\end{array}
$$
mound.

Charcoal from within material of mound, dating construction of

\section{CAR-394. Sample 4}

$$
\mathbf{4 7 0 0} \pm \mathbf{8 0}
$$

Charcoal from Socket hole F44 of chamber upright.

\section{England}

\section{Seamer Carr series}

Samples are from $\operatorname{Tr}$ C IX on palynol and archaeol site excavated 1979 by T Schadler-Hall, Kingston-upon-Hull Mus, Humberside, on shores of late Devonian Lake Pickering, at Seamer Carr, $6 \mathrm{~km} \mathrm{~S} \mathrm{of} \mathrm{Scarborough,} \mathrm{N}$ Yorkshire (54 $13^{\prime} \mathrm{N}, 0^{\circ} 25^{\prime} \mathrm{W}$, Natl Grid Ref TA 034819). Coll and subm 1979 by E Cloutman.

CAR-195. SCW1

$$
\begin{array}{r}
9480 \pm 110 \\
\delta^{13} C=-30.0 \%
\end{array}
$$

Birch branch from coarse detritus mud directly beneath Bone Layer 2135. Dates Late V or Early VI pollen zone. 
CAR-196. SCB1

$$
\begin{array}{r}
9100 \pm 100 \\
\delta^{13} C=-24.2^{\%} \%
\end{array}
$$

Collagen from bones (Cervus elephas, Bos primigenius) id by J Clutton Brock, British Mus (Nat Hist), London, from detritus mud assoc with mineral erosion deposit and hazel nut. Dates Late V or Early VI pollen zone.

CAR-197. Sample 2157

$$
\begin{array}{r}
9260 \pm 90 \\
\delta^{13} C=-26.0 \% 0
\end{array}
$$

Charcoal from Mineral Deposit 25, alt $13 \mathrm{~m}$, assoc with flint layer on shelf at lake edge.

\section{Scotland}

\section{Scord of Brouster series}

Charcoal samples are from settlement site comprising three houses, cairn, and surrounding irregular fields, excavated 1977 to 1979 by A W R Whittle, Dept Archaeol, Univ Coll, Cardiff, at Scord of Brouster, $25 \mathrm{~km} \mathrm{~N}$ of Lerwick, Shetland Isles $\left(60^{\circ} 15^{\prime} \mathrm{N}, 1^{\circ} 32^{\prime} \mathrm{W}\right.$, Natl Grid Ref HU 255516). Site, one of series of similar settlements, lies at head of sea inlet on $\mathrm{W}$ mainland of Shetland (Calder, 1958; Whittle, 1979). Site was excavated to examine chronology, subsistence, and environment and dated to examine chronol development at site. Coll 1977 to 1979 and subm 1980 by AWRW. Sample id by G Hillman.

CAR-242. Sample SB4

$$
\begin{array}{r}
4220 \pm 80 \\
\delta^{13} C=-26.0 \%
\end{array}
$$

Charcoal (Betula) from lower part of kerb cairn, Site IV. Dates construction of inner part of kerb cairn, in primary use as clearance cairn.

CAR-243. Sample SB5

$4100 \pm 70$

$$
\delta^{13} C=-25.7 \%
$$

Charcoal (Betula, Corylus) from occupation layer preceding construction of oval stone house, House 1, Phase 1, Tr D/E, Layer 4.

CAR-244. Sample SB6

$4460 \pm 70$

$\delta^{13} \mathrm{C}=-25.5 \%$ Phase 1.

Charcoal (Betula) from pre-house feature in $\mathrm{E}$ interior of House 1,

CAR-245. Sample SB7

$$
\begin{array}{r}
4350 \pm 90 \\
\delta^{13} C=-25.9 \%
\end{array}
$$

Charcoal (mostly Betula) from pre-house occupation layer beneath House 1, Phase 1, Layer 4.

\section{CAR-246. Sample SB8}

$$
\begin{array}{r}
\mathbf{4 0 5 0} \pm \mathbf{7 0} \\
\delta^{13} C=-26.1 \% 0
\end{array}
$$

Charcoal (Betula) from core of wall of oval stone house in $\operatorname{Tr} \mathrm{A}$ and C, House 1, Phase 2. Dates construction of House 1. 
CAR-247. Sample SB9

$4130 \pm 80$ $\delta^{13} C=-25.9 \%$

Charcoal (some Betula) from lowest level of Layer 3 at $\mathrm{N}$ half of interior of House 1, Phase 2, dating onset of use of house.

CAR-248. Sample SB10

$$
\begin{array}{r}
3670 \pm 80 \\
\delta^{13} C=-26.9 \% 0
\end{array}
$$

Charcoal from base of Layer 3 in $\mathrm{N}$ half of interior of House 1, Phase 2 , dating main phase of occupation of house.

CAR-249. Sample SB11

$$
\begin{array}{r}
4500 \pm \mathbf{8 0} \\
\delta^{13} C=-25.2 \% 0
\end{array}
$$

Charcoal (Betula, Corylus) from base of layer beneath wall on NW side of House 2, Phase 1, dating pre-house occupation.

\section{CAR-250. Sample SB12}

$$
\begin{array}{r}
4460 \pm 70 \\
\delta^{13} C=-25.7 \% 0
\end{array}
$$

Charcoal (Betula with some Corylus) from layer beneath wall, above Sample SB11, House 2, Phase 1. With CAR-249, should date length of prehouse occupation.

\section{CAR-251. Sample SB13}

$4540 \pm 70$

$\delta^{13} C=-24.6 \%$

Charcoal (Betula, Corylus and some blistered 6-row naked barley, Hardeum nudeum) from concentration in Layer 3d, in interior of House 2, Phase 1.

\section{CAR-252. Sample SB14}

$$
\begin{array}{r}
\mathbf{4 3 9 0} \pm \mathbf{8 0} \\
\delta^{13} C=-25.6 \% 0
\end{array}
$$

Charred fragments of Erica, Calluna plus grass stem and rhizomes and occasional cereal grains from within wall on NW side of House 2, Phase 2, dating construction of house.

\section{CAR-253. Sample SB15}

$$
\delta^{13} C=-26.3 \%
$$

Charcoal (Betula and Corylus) from Layer 2 within House 2, Phase 2, dating use of house.

\section{CAR-477. Sample SB16}

$$
\begin{array}{r}
3320 \pm 60 \\
\delta^{13} C=-24.9 \% o
\end{array}
$$

Charcoal and charred grain from W end of floor of House 1, Phase 1, dating main occupation of structure.

\section{CAR-479. Sample SB17}

$3420 \pm 70$

$\delta^{13} C=-24.8 \%$

Charcoal and charred grain from middle and $\mathrm{W}$ part of floor of House 3 , Phase 1, dating occupation of structure. 
General Comment (AWRW): series is within expected date range. CAR-253 is out of sequence. House 2 would appear to be slightly older than House 1 . CAR-246 and -247 are compatible with earlier determination (HAR-2413: $4170 \pm 80$ ) from House 1, Phase 2.

\section{REFERENCES}

Arnow, M, 1977, Noise discrimination method for pulse counting applications: Rev Sci Instruments, v 48, no. 10, p 1354-1355.

Boon, G C, 1967, Roman pottery from the base of the alluvium at Magor: Monmouthshire Antiquary, v 2, p 121-127.

Briggs, C S, 1975, A prehistoric complex on Cefn Gwernffrwd, Carmarthenshire: Archaeol Cambrensis, v 124, p 111-113.

Britnell, W J, 1979, The Gwernvale long cairn, Powys: Antiquity, v 53, p 132-134.

1980, Radiocarbon dates from the Gwernvale chambered tomb, Crickhowell, Powys: Antiquity, v 54, p 147.

1981, Trelystan: Current Archaeol, v 7, no. 7, p 201-205.

1982, The excavation of two round barrows at Trelystan, Powys: Prehist Soc Proc, $v$ 48, p 133-201.

Calder, C S T, 1958, Report on the discovery of numerous stone age house-sites in Shetland: Soc Antiquities Scotland Proc, v 89, p 340-397.

Chambers, F M, (ms), 1980, Aspects of vegetational history and blanket peat initiation in upland South Wales: PhD dissert, Univ Coll, Cardiff. letter, no. 35, November, p 24-29.

in South Wales: Jour Ecol, $v 70$, p 445-459. Wales: New Phytol, v 92, p 607-615.

1983a, New applications of palaeoecological techniques: integrating evidence of arable activity in pollen peat and soil stratigraphies, Cefn Graeanog, $\mathrm{N}$ Wales, in Jones, $\mathrm{M}$, ed, Integrating the subsistence economy: Assoc Environmental Archaeol no. 4, British Archaeol Repts, Internatl series 181, p 107-122.

$1983 \mathrm{~b}$, Three radiocarbon dated pollen diagrams from upland peats NW of Merthyr Tydfil, South Wales: Jour Ecol, v 71, no. 2, p 475-487.

$1983 \mathrm{c}$, The palaeoecological setting of Cefn Gwernffrwd- a prehistoric complex in Mid-Wales: Prehist Soc Proc, v 49, p 303-316.

Chambers, F M, Dresser, P Q, and Smith, A G, 1979, Radiocarbon dating evidence on the impact of atmospheric pollution on upland peats: Nature, $v 282$, no. 5741, p 829-831.

Cloutman, E C, (ms), 1983, Studies in the vegetational history of the Black Mountain region, South Wales: PhD dissert, Univ Coll, Cardiff.

Crew, P, 1978, Corn du, Brecon Beacons: Archaeol in Wales, no. 18, p 31.

Dowdell, G, 1978, Magor, Gwent: Swansea, Glamorgan Gwent Archaeol Trust ann rept, $1977-1978, \mathrm{p} 23$.

Dresser, P Q, (ms), 1970, A study of sampling and pretreatments of materials for radiocarbon dating: PhD dissert, Queen's Univ, Belfast.

Gardner, W and Savory, H N, 1964, Dinorben: Natl Mus Wales, Cardiff.

Guilbert, G, 1979, Dinorben 1977-8: Current Archaeol, v 6, no. 6, p 182-188.

1980a, Dinorben radiocarbon dates: Current Archaeol, v 6, no. 11, p 336-338. 1980b, Dinorben: Archaeol in Wales 1979, v 19, p 14.

Jones, R, Benson-Evans, K, Chambers, F M, Abell Seddon, B, and Yuk Chun Tai, 1978, Biological and chemical studies of sediments from Llangorse lake, Wales: Verh Internatl Verein Limnol, v 20, p 642-648.

Kelly, R S, 1982, The excavation of a medieval farmstead at Cefn Graeanog, Clynog, Gwynedd: Board Celtic Studies Bull, v 29, no. 4, p 858-907.

Lynch, F M, in press a, Excavation of Moel Goedog Circle I: a complex Ring Cairn near Harlech, Merioneth: Archeol Cambrensis, v 133, in press. eth: Archeol Cambrensis, $v 134$, in press.

Mitchell, G F, 1956, Post boreal pollen diagrams from Irish raised bogs: Royal Irish Acad Proc, Sec B, v 57, p 185-251.

Morgan, J G and Ruggles, C, 1976, Indications at the Cefn Gwernffrwd site: Archaeol Cambrensis, v 125 , p 162-165.

Otlet, R L, Walker, A J, Hewson, A D, and Burleigh, R, $1980,{ }^{14} \mathrm{C}$ interlaboratory comparison 
in the UK: experiment design, preparation and preliminary results, in Stuiver, Minze and Kra, Renee, eds, Internatl radiocarbon conf, 10th, Proc: Radiocarbon, v 22, no. 3, p 936946.

Pennington, W, 1977, The late Devonian flora and vegetation of Britain: Royal Soc [London] Philos Trans, Sec B, v 280, p 247-271.

Savory, H N, 1971, Excavations at Dinorben 1965-9: Natl Mus Wales, Cardiff.

Switsur, V R, 1972, Combustion bombs for radiocarbon dating, in Rafter, T A, and GrantTaylor, T, eds, Internatl radiocarbon conf, 8th, Proc, Wellington, New Zealand, Royal Soc New Zealand, v 1, p 120-132.

Whittle, A, 1979, Scord of Brouster: Current Archaeol, v 6, no. 6, p 167-171. 